\title{
Fast Clock Scheduling and an Application to Clock Tree Synthesis
}

\author{
Rickard Ewetz* and Cheng-Kok Koh \\ School of Electrical and Computer Engineering, Purdue University \\ Corresponding author: Rickard Ewetz \\ address: 1285 Electrical Engineering Building, West Lafayette, IN 47907-2035. \\ phone: +1-765-637-6672, fax: +1-765-494-6951 \\ rewetz,chengkok@purdue.edu
}

\begin{abstract}
Clock networks are required to be constructed with adequate safety margins in the skew constraints to operate correctly even under the influence of variations. In this work, a scalable clock scheduler is developed to drive a synthesis framework that constructs useful skew clock trees with large safety margins that are tailored to the tree topology. Sequential elements are clustered early in the topology, if it is impossible provide adequate robustness to variations using only safety margins. Compared to earlier studies, the proposed framework performs the clock scheduling one to two orders of magnitude faster and improves yield and capacitive cost on several synthesized circuits.
\end{abstract}

\section{Keywords}

Algorithms, clock network synthesis, low-power, physical design, skew

\section{INTRODUCTION}

In the synthesis of clock networks for modern VLSI circuits, it is becoming more important to exploit the non-uniformity of the slacks in the timing constraints as process, voltage and temperature variations continue to diminish the available timing margins. The clock network of a VLSI circuit is constructed after an initial placement and delay estimation of the data and control paths. Skew is the difference of the arrival time of the clock signal between a pair of sequential elements. The clock network must meet setup and hold time constraints (i.e., skew constraints) imposed by the data and control paths. Moreover, the skew constraints must be satisfied even when the clock network is under the influence of variations. Such a problem is the emphasis of two clock contests organized by the International Symposium on Physical Design in 2009 [3] and 2010 [4]. However, the two contests considered uniform and regular skew constraints. In reality, the slacks in the skew

\footnotetext{
*Present address: 4328 Scorpius St., ECE Dept., University of Central Florida, Orlando, FL 32816.
}

constraints imposed by the data and control paths are typically non-uniform and it is necessary to use useful skew to obtain high yield.

The Greedy-useful skew tree/deferred-merge embedding (Greedy-UST/DME) algorithm was proposed to construct clock trees meeting arbitrary skew constraints [1]. The algorithm is based on integrating tree construction and dynamic clock scheduling. Clock scheduling is the process of specifying the arrival time of the clock signal to the sequential elements, or the clock sinks. In dynamic clock scheduling, a clock schedule is incrementally specified by committing the skew between pairs of sequential elements. It was shown that if and only if each skew is committed within an feasible skew range (FSR), the skew constraints are satisfied. In the Greedy-UST/DME algorithm, the skew constraints are captured in a skew constraint graph (SCG) and a clock tree is constructed by joining subtrees pair-wise to form larger subtrees. Moreover, subtrees are joined such that the skew of the each subtree-pair is committed within the FSR of the pair, and the FSRs are found by computing shortest paths in the SCG.

To satisfy skew constraints under variations, safety margins can be inserted in the skew constraints. In [5], it was shown that there exists a maximum uniform safety margin $M$ that can be inserted uniformly in the skew constraints. In [1], a user specified uniform safety margin $M_{\text {user }} \leq M$ was inserted, which resulted in a smooth trade-off between capacitive cost and robustness to variations. In [2], safety margins of different magnitudes were inserted in different skew constraints during the construction of a clock tree. Smaller safety margins were inserted closer to the bottom of the tree and larger safety margins were attempted to be inserted closer to the top of the tree. The drawbacks of these works are that the dynamic clock scheduling is not very scalable (practically limited to designs with a few thousand sequential elements) and yield loss may be suffered due to inadequate safety margins.

We propose a framework to construct useful skew clock trees with large tailored safety margins (UST- 
LTSM), driven by a scalable clock scheduler. The main innovations of the framework are: (1) A dynamic clock scheduler that is based on sparse-graph algorithms. (2) Robustness to variations is provided by combining the use of safety margins and placing certain sequential elements close in the tree topology to lower the point of divergence. (3) The inserted safety margins are tailored to the tree topology by reducing safety margins that are larger than required.

Compared with the clock scheduler in [1], our proposed clock scheduler utilizes that the SCG is sparse, which results better scalability. We overcome the limitation of $M_{\text {user }} \leq M$ in [1]. Specifically, we ensure that the full safety margin $M_{\text {user }}$ is provided in a majority of the skew constraints and in skew constraints were it is impossible to provide a safety margin $M_{\text {user }}$, a safety margin of at least $M$ is provided and the corresponding sequential elements are clustered early in the tree construction process. Compared with the tailoring of safety margins in [2], our tailoring techniques can never "run-out" of safety margin at the top of the clock tree. The techniques are also scalable as they are based on the developed dynamic clock scheduler. Moreover, we avoid constructing tree topologies where an adequate safety margin can never be provided in certain tight skew constraints, as is illustrated in Figure 1.

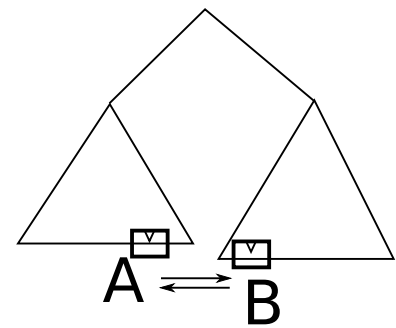

(a)

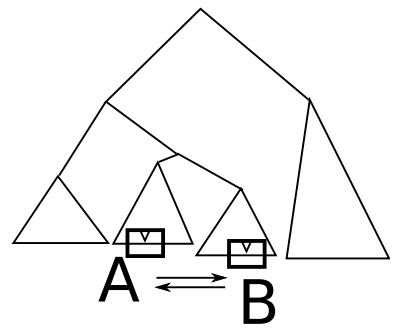

(b)
Figure 1: Sequential elements $A$ and $B$ have tight skew constraints. If $A$ and $B$ are distant in the topology, as in (a), the safety margin may be inadequate. The two elements should be placed closer, as in (b), in the topology.

The experimental results show that the proposed clock scheduler is one to two orders of magnitude faster compared with schedulers in previous studies. On a benchmark circuit with 13, 216 sequential elements, the framework provides a yield of $97 \%$.

The remainder of the paper is organized as follows: the problem formulation is introduced in Section 2 and previous studies are reviewed in Section 3. An overview of the proposed dynamic clock scheduling and UST-LTSM framework is presented in Section 4. The details of dynamic clock scheduling and the UST-LTSM framework are given in Section 5 and Section 6, respectively. Finally, the experimental results are explained in Section 7 and the paper is concluded in Section 8 .

\section{PROBLEM FORMULATION}

This paper considers the problem of constructing a variations aware useful skew clock tree (VA-UST). For a clock tree to be able to satisfy timing constraints while under the influence of variations, safety margins are required to be provided in the skew constraints. Therefore, we present the setup and hold time constraints and the insertion of safety margins before introducing the VA-UST synthesis problem. Next, we present the dynamic clock scheduling problem because it is a key component of constructing useful skew clock trees.

\subsection{Skew constraints and safety margins}

Setup and hold time constraints are imposed between flip flops (FFs) that are only separated by combinational logic. A launching and a capturing flip flop (denoted as $\mathrm{FF}_{i}$ and $\mathrm{FF}_{j}$, respectively) are illustrated in Figure 2(a) and the setup and hold time constraints of the flip flop pair are formulated as follows:

$$
\begin{aligned}
t_{i}+t_{i}^{C Q}+t_{i j}^{\max }+t_{j}^{S} & \leq t_{j}+T, \\
t_{i}+t_{i}^{C Q}+t_{i j}^{\text {min }} & \geq t_{j}+t_{j}^{H} .
\end{aligned}
$$

Here, $t_{i}$ and $t_{j}$ are respectively the arrival times of the clock signal to $\mathrm{FF}_{i}$ and $\mathrm{FF}_{j} ; t_{i}^{C Q}$ is the clock to output time of $\mathrm{FF}_{i} ; t_{i j}^{\max }$ and $t_{i j}^{\min }$ are the maximum and minimum propagation delay, respectively; $t_{j}^{S}$ and $t_{j}^{H}$ are the setup and hold time of $\mathrm{FF}_{j}$, respectively. The constraints in Eq. (1) and Eq. (2) are reformulated with respect to the skew skew $w_{i j}=t_{i}-t_{j}$ using $l_{i j}=t_{j}^{H}-t_{i}^{C Q}-t_{i j}^{\min }$ and $u_{i j}=T-t_{i}^{C Q}-t_{i j}^{\max }-t_{i}^{S}$ to obtain

$$
l_{i j} \leq \operatorname{skew}_{i j} \leq u_{i j}
$$

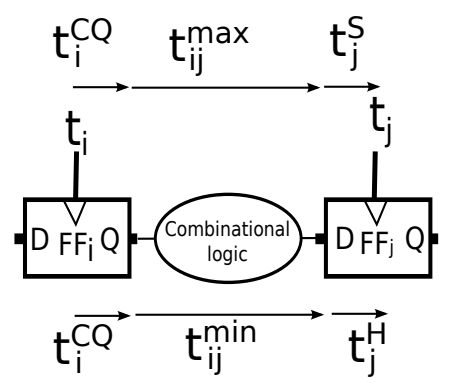

(a)

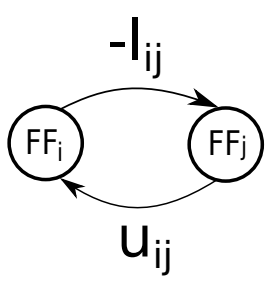

(b)
Figure 2: (a) Two flip flop circuit. (b) SCG of the two flip flop circuit in (a).

The slacks in the lower and upper bound of the skew constraint are formulated as skewij $-l_{i j}$ and $u_{i j}$ skew $_{i j}$, respectively. The skew constraints in Eq. (3) can be captured in a skew constraint graph (SCG). In an SCG, $G=(V, E)$, the vertices $V$ represent the flip flops and the edges $E$ represent the skew constraints. A directed edge $e_{i j}$ with a weight $w_{i j}=-l_{i j}$ is added 
from vertex $i$ to vertex $j$ and a directed edge $e_{j i}$ with a weight $w_{j i}=u_{i j}$ is added from vertex $j$ to $i$. In Figure 2(b), the SCG of the two flip flop circuit in Figure 2(a) is shown. A feasible clock schedule exists if the SCG does not contain any negative cycles. Moreover, one such feasible clock schedule can be determined using the Bellman-Ford algorithm [6].

To provide a (non-negative) safety margin to variations, the skew constraints in Eq. (3) can be tightened with non-uniform safety margins $m_{j i}$ and $m_{i j}$, respectively, to obtain:

$$
l_{i j}+m_{j i} \leq \text { skew }_{i j} \leq u_{i j}-m_{i j}
$$

Note that inserting an safety margin in a skew constraint corresponds to reducing the weight of an edge in the SCG, which may create a negative cycle.

\subsection{Variations-aware useful skew clock tree (VA-UST) problem}

The VA-UST problem is based on an adaptation of the problem formulation in the International Symposium on Physical Design 2010 contest [4]. The main difference is that we consider arbitrary skew constraints.

The problem consists of constructing a clock tree that delivers a synchronizing clock signal from a clock source to a set of clock sinks. The source-to-sink connections are realized using wires and buffers from a wire library and a buffer library, respectively. The clock tree is to be constructed such that the clock signal is delivered meeting the skew constraints specified in Eq. (3), while under the influence of variations. The key idea is to construct a clock tree with adequate safety margins provided in the skew constraints, as detailed in Eq. (4). In particular, the safety margins $m_{i j}$ and $m_{j i}$ in the skew constraints between $\mathrm{FF}_{i}$ and $\mathrm{FF}_{j}$ must be larger than the maximum skew that can be caused by variations between the two flip flops. (The details of the Monte Carlo framework that is used to evaluate the timing performance under variations is given in Section 7.1.)

Moreover, to ensure sharp rise and fall times, the clock network must also satisfy a transition time constraint. Transition time is the $10 \%-90 \%$ rise or fall time of the clock signal and the transition time at every point in the clock network must be less than a constraint parameter $S_{\text {tran }}$.

\subsection{Dynamic clock scheduling problem}

A related problem to the VA-UST problem is the dynamic clock scheduling problem. A clock schedule is a set of relative arrival times of the clock signal to the clock sinks. In a bottom-up clock synthesis process, the clock schedule is determined by performing $|V|-1$ incremental skew commitments in an SCG iteratively. Before each skew commitment is made between two flip flops (or subtrees) a feasible skew range is computed. The skew commitment then narrows the FSR to a specific skew value, necessitating an operation to update the $S C G$. Moreover, to guide the synthesis process, FSRs of subtree-pairs that are spatially close, or nearest neighbors, are required to be computed, which is performed using an compute nearest-neighbors' (NN) feasible skew ranges operation. We define the dynamic clock skew scheduling problem to be the problem of performing the following operations:

The compute-FSR operation calculates the $\mathrm{FSR}_{i j}=$ $\left[-d_{i j}, d_{j i}\right]$ between two subtrees $i$ and $j$, where $d_{i j}$ and $d_{j i}$ denote the shortest paths in the SCG from vertex $i$ to vertex $j$ and from $j$ to $i$, respectively. If we commit the skew between $i$ and $j$, denoted $s k e w_{i j}$, to be within $\mathrm{FSR}_{i j}$, a feasible clock schedule exists.

The compute-NN-FSRs operation computes FSRs of subtree-pairs that are spatially close. Let $k$ be the number of subtree-pairs that are spatially close. Some schedulers can perform a compute-NN-FSRs operation to obtain all $k$ FSRs faster than performing a series of $k$ compute-FSR operations.

The update-SCG operation takes place when we commit the skew between two vertices, skew $w_{i j}=t_{i}-t_{j}$, from an FSR to a particular value, say $a$. An edge $e_{j i}$, with weight $w_{j i}=a$ is added to the SCG, and an edge $e_{i j}$, with weight $w_{i j}=-a$ is added. The edges ensures that, $t_{i}-t_{j} \leq a$ and $t_{i}-t_{j} \geq a$, or equivalently $s k e w_{i j}=t_{i}-t_{j}=a$. Note that the added edges are always a reduction of the existing edge weights (nonexisting edge can be considered to have a edge weight of $\infty[1])$.

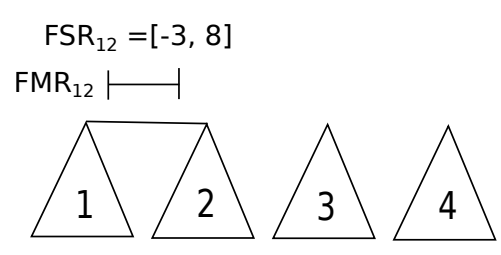

(a)

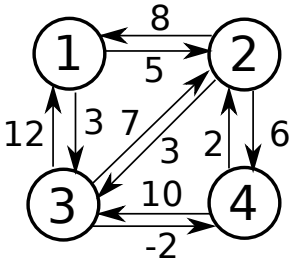

(b)

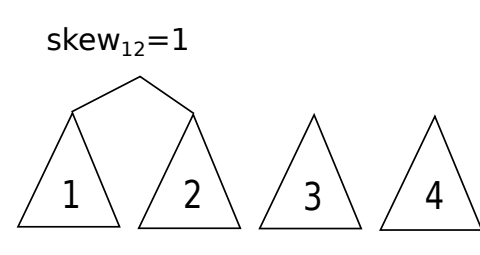

(c)

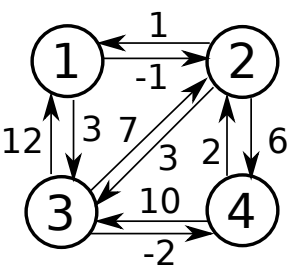

(d)
Figure 3: (a) To join the subtrees 1 and 2, the $\mathbf{F S R}_{12}$ is computed and the $\mathbf{F M R}_{12}$ is constructed. (b) An SCG. (c) The subtrees 1 and 2 are joined physically. (d) The SCG is updated with the skew commitment $s k e w_{12}=1$.

\section{PREVIOUS WORK}

In this section, we review the Greedy-UST/DME algorithm [1], the dynamic clock scheduling in [1], and the insertion of safety margins in [1] and [2]. A list with abbreviations that are used is given in Table 1. 


\begin{tabular}{|c|l|}
\hline UST & Useful skew tree \\
DME & Deferred-merge embedding \\
SCG & Skew constraint graph \\
APSP & All-pairs shortest paths \\
SSSP & Single source shortest paths \\
FSR & Feasible skew range \\
FMR & Feasible merging range \\
NNG & Nearest neighbor graph \\
NN-FSRs & Nearest neighbors' feasible skew ranges \\
VS & Virtual source \\
VSDV & Virtual source distance vector \\
\hline
\end{tabular}

Table 1: List of abbreviations.

\subsection{The Greedy-UST/DME algorithm}

The Greedy-UST/DME algorithm constructs a useful skew tree (UST) that satisfies a set of arbitrary skew constraints. An overview of the algorithm is shown in Algorithm 1 and the calls to the clock scheduler are shown in bold. The algorithm is illustrated with an example shown in Figure 3.

The Greedy-UST/DME algorithm is based on a bottomup synthesis process in which a clock tree is constructed by iteratively joining smaller subtrees to form larger subtrees. The subtrees are placed in a nearest neighbor graph (NNG) and the skew constraints are captured in an SCG. In an NNG, the distance between two subtrees is defined to be the wiring cost of merging that pair of subtrees into a larger subtree. The wiring cost of merging two subtrees depends on the spatial distance, the timing, and the FSR of the subtree-pair. To compute the distance between different pairs of subtrees in the NNG, the compute-NN-FSRs operation is used to compute the required FSRs, which is based on the constraints in the SCG. Iteratively, the subtree-pairs that are closest in the NNG are selected to be merged. Consider merging the subtrees 1 and 2 in Figure 3(a), with the SCG in Figure 3(b). First, the FSR $_{12}$ between the two subtrees is computed using the compute-FSR operation. Next, the $\mathrm{FSR}_{12}$ is converted into a feasible merging range (FMR). Since the physically joining location of a subtree-pair determines the skew of the pair, the $\mathrm{FMR}_{12}$ is constructed such that if the subtree-pair is joined physically within the $\mathrm{FMR}_{12}$, the skew of the subtree-pair will be committed within the FSR $\mathrm{F}_{12}$. (The details of how an FSR is converted to an FMR based on the Elmore delay model is provided in [1]). From the SCG in Figure 3(b), $\mathrm{FSR}_{12}=\left[-d_{12}, d_{21}\right]=[-3,8]$ and the corresponding $\mathrm{FMR}_{12}$ is shown in Figure 3(a). In Figure 3(c), the selected merging location (within the $\mathrm{FMR}_{12}$ ) happens to result in a skew commitment of skew $_{12}=1$ (guaranteed to be within the $\mathrm{FSR}_{12}$ ). Each skew commitment is equivalent to adding (or updating) two edges in the SCG, which is performed using the update-SCG operation. In Figure 3(d), the edge weights of the edges $e_{12}$ and $e_{21}$ are updated to $w_{12}=-1$ and $w_{21}=1$, respectively. Next, the merging process in the
NNG is iterated until the remaining three subtrees have been joined to a single clock tree.

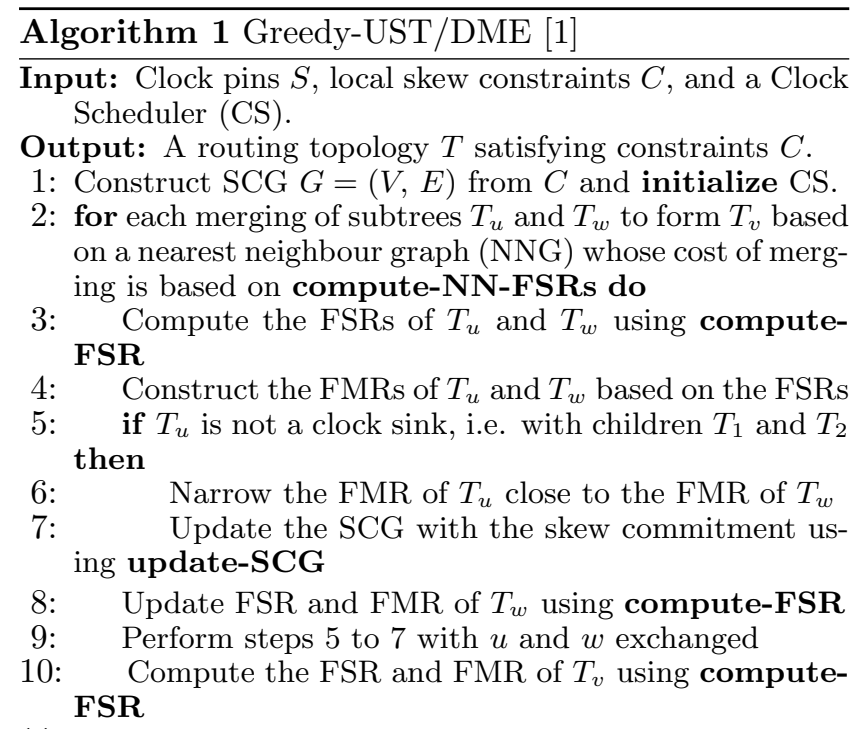

11: Perform DME top-down embedding.

12: return: $\mathrm{T}$

\subsection{Clock scheduling using the scheduler in [1]}

The scheduler in [1] is based on the Floyd-Warshall all-pairs shortest paths (APSP) algorithm [6]. Throughout the synthesis process an APSP matrix is maintained, storing all the shortest paths of the SCG. We refer to this scheduler as a scheduler with memory (M-Scheduler) because an APSP matrix is used.

The M-scheduler first initializes the APSP matrix using the Floyd-Warshall algorithm in $O\left(V^{3}\right)$ [6]. Using the APSP matrix the M-scheduler can perform Compute-FSR operations, Compute-NN-FSRs operations, and update-SCG operations in $O(1), O(k)$, and $O\left(V^{2}\right)$, respectively.

Table 2: Greedy-UST/DME run-times. $t_{t o t}$ denotes the total run-time and $t_{s}$ is the run-time of the scheduler. A ' $-(\mathrm{T})$ ' or ' $-(\mathrm{M})$ ' means that the run-time is more than 15 hours, and the memory is insufficient, respectively.

\begin{tabular}{|c|c|c|c|c|}
\hline \multirow[t]{2}{*}{ Circuit } & \multirow{2}{*}{$\begin{array}{l}\text { Sinks } \\
\text { (num) }\end{array}$} & \multirow{2}{*}{$\begin{array}{c}\text { Skew } \\
\text { constraints } \\
\text { (num) }\end{array}$} & \multicolumn{2}{|c|}{ M-Scheduler } \\
\hline & & & $\begin{array}{c}t_{t o t} \\
(\mathrm{~s})\end{array}$ & $\begin{array}{l}t_{s} \\
(\mathrm{~s})\end{array}$ \\
\hline D-1 & 1000 & 6000 & 18 & 17 \\
\hline D-2 & 2000 & 12000 & 188 & 187 \\
\hline D-3 & 5000 & 30000 & 2524 & 2519 \\
\hline D-4 & 10000 & 60000 & 16725 & 16698 \\
\hline D-5 & 20000 & 120000 & $-(\mathrm{T})$ & $-(\mathrm{T})$ \\
\hline D-6 & 50000 & 300000 & $-(\mathrm{M})$ & $-(\mathrm{M})$ \\
\hline D-7 & 100000 & 600000 & $-(\mathrm{M})$ & $-(\mathrm{M})$ \\
\hline
\end{tabular}

In Table 2, we show the run-time of the GreedyUST/DME application and the portion of the run-time 
that is consumed by the clock scheduler in the columns labeled $t_{t o t}$ and $t_{s}$, respectively. (The details of the benchmark circuits and the experimental setup are provided in Section 5.3.) Based on the results in the table, it is observed that a majority of the total run-time of the application is consumed by the scheduler and that the run-time consumed by the scheduler grows rapidly when the number of sinks increases. The scalability of the M-scheduler is limited because the run-time complexity of the update-SCG operation is $O\left(V^{2}\right)$ and it is performed $V-1$ times, yielding a total run-time complexity of $O\left(V^{3}\right)$. The computation on the initial APSP matrix using Floyd-Warshall is also $O\left(V^{3}\right)$. Naturally, the memory requirement is $O\left(V^{2}\right)$ as an APSP matrix is used, which restricts the number of sinks to be less than 50,000 (under our experimental setup).

\subsection{Insertion of safety margins in [1] and [2]}

The amount of safety margin that can be inserted in a single skew constraint is not limited by the skew constraint itself. The arrival time between the two sinks of a skew constraint can be skewed arbitrarily, to create a large safety margin. It is the total amount of safety margin that can be inserted in every cycle of skew constraints that is limited, as explained below.

Consider an arbitrary cycle $C=\left(v_{1}, v_{2}\right),\left(v_{2}, v_{3}\right), \ldots$, $\left(v_{n-1}, v_{n}\right),\left(v_{n}, v_{1}\right)$, where $v_{i}, 1 \leq i \leq n$, are vertices in the SCG for Eq. (4). Each edge in the SCG represents an inequality constraint $t_{i}-t_{j} \leq w_{j i}-m_{i j}$. Let $|C|=$ $n$ be the number of edges in the cycle $C$ and $W_{C}=$ $\sum_{(i, j) \in C} w_{j i}$. By summing the inequality constraints corresponding to a cycle $C$, we obtain Eq. (5), which can be reformulated into Eq. (6).

$$
\begin{aligned}
\sum_{(i, j) \in C}\left(t_{i}-t_{j}\right) & \leq \sum_{(i, j) \in C}\left(w_{j i}-m_{i j}\right), \\
\sum_{(i, j) \in C} m_{i j} & \leq W_{C} .
\end{aligned}
$$

Eq. (6) specifies that the maximum safety margin that can be allocated across a cycle in total is at most $W_{C}$. The ratio $W_{C} /|C|$ is defined to the the average margin $M_{C}$ of a cycle. We also define the maximum margin $M_{i j}^{\max }$ of an edge $e_{i j}$ as follows: Consider all cycles that the edge $e_{i j}$ participates in. The $M_{i j}^{\max }$ of the edge $e_{i j}$ is the minimum of the average margin of all these cycles. Note that the minimum of the maximum margins of all edges is the maximum uniform safety margin $M$ in [5]. We exemplify these notations using the SCG in Figure 3(b). Let $C_{t}$ denote a specific cycle and in particular let $C_{1}=(2,3),(3,4),(4,2)$. For the cycle $C_{1}$, $W_{C_{1}}=3$ and $M_{C_{1}}=1$. Moreover, since the cycle $C_{1}$ is the cycle with the smallest average margin of all the cycles in the SCG, the maximum uniform safety margin $M=1$.

In [1], a user specified safety margin $M_{\text {user }}$ was imposed uniformly on all the skew constraints. A smooth trade-off between cost and robustness was obtained for different values of the safety margin $M_{\text {user }}$. A limitation is that the user specified safety margin $M_{u s e r}$ is required to be no greater than the maximum uniform safety margin $M$ in [5], or negative cycles are created in the SCG, which yields an infeasible clock schedule.

The case of $M_{\text {user }}>M$ is of particular interest for modern designs with tight skew constraints. In [2], the problem of providing such large safety margins was considered. In that work, the safety margins were dynamically added during the bottom-up process of constructing a clock tree. In the synthesis process, which consists of iterative merging of subtrees, the required safety margin between two subtrees is computed and inserted when two subtrees are joined to form larger subtree. Effectively, the approach inserts small safety margins at the bottom of the topology and large safety margins near the root node. In a sense, the algorithm nonuniformly distributes the total available margin $M_{C}$ in each cycle in the SCG such that smaller safety margins are inserted at the bottom of the tree, potentially leaving larger margins for the top of the tree.

The first drawback of the approach is that all the safety margins may be completely utilized at the bottom of the clock tree, leaving none at the top, where it is needed the most. The second drawback is that for some pair of sinks, it may not be possible to insert a safety margin greater than $M$. Consider a cycle $C$ with an average margin of $M$ and $|C|=2$. As the cycle is of length 2 , it is not possible to skew the margin in the cycle. Therefore, the maximum safety margin between the pair of sinks will be $M$, no matter how the other safety margins are inserted in the tree. The algorithm in [2] did not consider this aspect of the problem and could therefore not guarantee that such sink pairs be joined with adequate safety margins. In particular, if the sink pair is joined at the root of the clock tree, as shown in Figure 1(a), the available safety margin may not be sufficient.

Based on this argument we conclude that to ensure safety margins $M_{\text {user }}>M$ at the root of the tree topology the problem of inserting safety margins is not separable from the choice of the tree topology.

\section{PROPOSED SCHEDULING AND SYN- THESIS}

\subsection{Proposed clock scheduling}

We propose an on-the-fly sparse-graph-based clock scheduler that computes shortest paths when they are requested by a synthesis application. The computation of the shortest paths is performed using Dijkstra's shortest path algorithm and the alternative edge weights used in Johnson's algorithm. The alternate edge weights (which are all positive) are required to be introduced because Dijkstra's algorithm cannot be applied to graphs with negative edge weights. The alternative edge weights are defined by an auxiliary vector $h$ that stores a sin- 
gle source shortest path (SSSP) solution from a virtual source to each of the vertices in the SCG, yielding a memory requirement of $O(V)$.

When a skew commitment is made, the auxiliary vector $h$ is required to be updated. The update is performed using a incremental update that is similar to Dijkstra's algorithm.

\subsection{Proposed UST-LTSM framework}

We propose the UST-LTSM framework driven by the on-the-fly sparse-graph scheduler. The framework is designed to satisfy all skew constraints where the skew caused by variations is less than $M_{\text {user }}$.

The main idea is to divide edges in the SCG (or skew constraints) into loose and tight edges (constraints). We refer to edges $e_{i j},(i, j) \in E$, with a maximum margin of $M_{i j}^{\text {max }}<M_{\text {user }}$ as tight edges or tight skew constraints. In the pre-tree construction phase, a safety margin of $M_{\text {user }}$ is directly inserted in the loose skew constraints, which is a majority of the skew constraints. (These skew constraints do not require further consideration.) In the case of tight skew constraints, the full safety margin $M_{\text {user }}$ cannot be provided. Instead, a safety margin of at least $M$ is inserted. Moreover, the sinks corresponding to these skew constraints are clustered to ensure that they are placed close in the tree topology, as shown in Figure 1(b). It is well known that the variations-induced skew between a pair of sinks that are close in the topology is less than that of sinks that are distant in the tree because of a lower point of divergence. Note that the insertion is equivalent to the approach in [1] for $M_{\text {user }} \leq M$. However, in contrast to [1], we are not limited to $M_{\text {user }} \leq M$.

Furthermore, as topological commitments are made during the tree construction phase, the amount of safety margin that is required in certain skew constraints can be determined, allowing the inserted safety margins in these constraints to be reduced, or tailored to the tree topology. We propose two methods to perform such tailoring, either directly when two subtrees are merged, or by reconstructing subtrees. In contrast to earlier studies, both methods are based on incrementally reducing the inserted safety margin. Earlier studies are based on incrementally inserting safety margins. Note that the tailoring technique that is based on reconstructing subtrees requires additional FSRs to be computed, which is facilitated by the proposed dynamic clock scheduler. Without the developed clock scheduler, the approach would have had longer run-time.

\section{DYNAMIC CLOCK SCHEDULING}

In this section we propose an on-the-fly sparse-graphbased scheduler (FS-scheduler), which computes a series of shortest paths in an incrementally changing weighted graph. ${ }^{1}$ The FS-scheduler is built based on the selec-

\footnotetext{
${ }^{1}$ We have also developed a sparse-graph based scheduler that uses an APSP matrix and it is presented in [7].
}

tion and combination of different existing shortest path algorithms and suitable auxiliary data structures $[6,8$, 9].

\subsection{The FS-scheduler}

The on-the-fly sparse-graph-based scheduler computes shortest paths in the SCG as they are requested by compute-FSR calls. To enable the use of the Dijkstra's algorithm [6] to compute requested shortest paths, the alternative edge weights in Johnson's algorithm [6] are used, which translates all the edge weights into nonnegative edge weights.

A virtual source (VS) that is connected to each of the vertices of an SCG with an virtual edge with a weight of 0 is introduced, which is illustrated in Figure 4(a). The auxiliary virtual source distance vector (VSDV) $h$ that stores the SSSPs from a virtual source to all the vertices of the SCG. Next, the alternative edge weights are defined as $\bar{w}_{i j}=w_{i j}+h_{i}-h_{j}, \forall e_{i j} \in E$, where $h_{i}$ is the shortest path from the virtual source to the vertex $i$ (stored in the VSDV $h$ ) and $\bar{w}_{i j}$ is the alternative nonnegative edge weight for the edge $e_{i j}$. The alternative edge weights for the SCG in Figure 4(a) are shown in Figure 4(b) (Note that in our implementation, only the original edge weights and the VSDV $h$ are stored and the alternative edge weights are defined implicitly.) The original shortest paths $d$ and the shortest paths defined by the alternative edge weights $\bar{d}$, satisfy the following relationship: $d_{i j}=\bar{d}_{i j}+h_{j}-h_{i}$. Consequently, a shortest path $d_{i j}$ can be determined by computing $\bar{d}_{i j}$ using Dijkstra's algorithm and the alternative edge weights. Next, $\bar{d}_{i j}$ is translated into $d_{i j}$ using the VSDV $h$ [6].

The VSDV $h$ is required to be maintained updated throughout the scheduling process. Initially, the auxiliary VSDV $h$ is initialized using the Bellman-Ford [6] algorithm in $O(V E)$.

Compute-FSR: A compute-FSR call requires the computation of two shortest paths. Dijkstra's algorithm is executed twice to compute these two shortest paths using the alternative edge weights defined by $h$, as shown in (c) and (d) of Figure 4. In addition, Dijkstra's algorithm is modified with an trivial early exit procedure. If the shortest path $d_{x y}$ is queried, the algorithm terminates after the vertex $y$ is extracted from the minimum priority queue $Q$ that Dijkstra's algorithm maintains.

The compute-FSR operation is based on Dijkstra's algorithm, which has a complexity of $O(V \log V+E)$ [6]. However, the early exit procedure may terminate the algorithm early. $E_{F S} \subseteq E$ and $V_{F S} \subseteq V$ are the edges and vertices visited before the early exit, as shown in (c) and (d) of Figure 4. The complexity of the computeFSR operation is $O\left(V_{F S} \log V+E_{F S}\right)$.

Compute-NN-FSRs: To guide the synthesis process in the selection of which subtrees that are to be merged, FSRs between subtrees that are spatially close to each other are required to be computed. Let there be $k$ FSRs that are required to be computed. A naive 


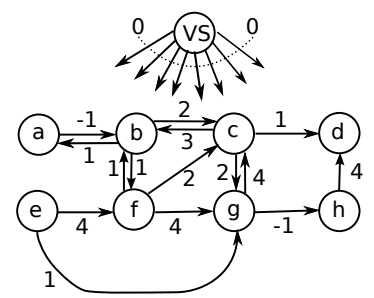

(a) An SCG with a VS

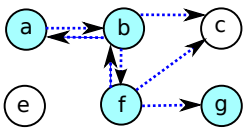

(c) Computing dag

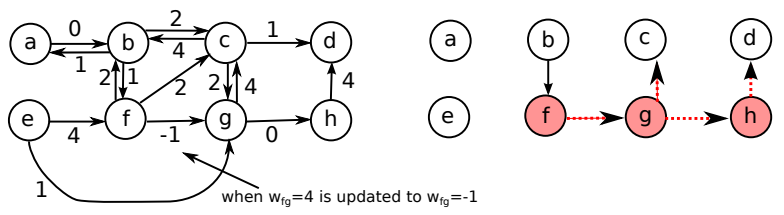

$\begin{array}{ll}\text { (e) Update of the weight } w_{f g} & \text { (f) Update of VSDV } h^{\text {OUT }} \text { to } h\end{array}$

\begin{tabular}{|c|cccccccc|}
\hline & $\mathrm{a}$ & $\mathrm{b}$ & $\mathrm{c}$ & $\mathrm{d}$ & $\mathrm{e}$ & $\mathrm{f}$ & $\mathrm{g}$ & $\mathrm{h}$ \\
\hline$h^{\text {OUT }}$ & 0 & -1 & 0 & 0 & 0 & 0 & 0 & -1 \\
$h$ & 0 & -1 & 0 & 0 & 0 & 0 & -1 & -2 \\
\hline
\end{tabular}

(g) Update of VSDV $h^{\text {OUT }}$ to VSDV $h$ using the traversal in (f)

Figure 4: (a) An SCG with a virtual source and virtual edges. The VSDV $h^{O U T}$ of the SCG in (a) is shown in (g). (b) The SCG in (a) is displayed with the alternative edge weights in Johnson's algorithm. In (c) and (d), Dijkstra's algorithm is used to compute the $\mathbf{F S R}_{a g}$. The vertices $V_{F S}$ and edges $E_{F S}$ are shown in blue. (e) The edge weight $w_{f g}=4$ is updated to $w_{f g}=-1$. (f) The outdated VSDV $h^{O U T}$ is updated to VSDV $h$. The traversed edges $E_{H}$ are shown in dashed red and visited vertices $V_{H}$ are shown in a solid light red.

method would be to perform $k$ compute-FSR calls in series, which leads to several executions of Dijkstra's algorithm from the same vertex. Instead we execute Dijkstra's algorithm at most once from each vertex and compute all the requested shortest paths from that source at the same time, before performing an early termination.

A FSR is computed with respect to two subtrees. Let $K$ be the set of subtrees which correspond to all the subtrees involved in the Compute-NN-FSRs operation and let $|K|$ be the number of subtrees in $K$ with $2 \leq$ $K \leq 2 k$. Then an execution of Dijkstra's algorithm is required from each subtree $s \in K$, each with a runtime complexity of $O\left(V_{F S K}^{s} \log V_{F S K}^{s}+E_{F S K}^{s}\right)$. Let $V_{F S K}=\cup_{s \in K} V_{F S K}^{s}$ and $E_{F S K}=\cup_{s \in K} E_{F S K}^{s}$ then the compute-NN-FSRs is $O\left(|K|\left(V_{F S K} \log V_{F S K}+E_{F S K}\right)\right)$. The memory requirement is $O(k)$ to store the computed FSRs.

Update-SCG: When two edges are added to the SCG, the VSDV $h$ may need to be updated. The VSDV $h$ is updated using the incremental SSSP update in Algorithm 2 [9].

The incremental update is based on Dijkstra's algorithm, the main difference is that the priority queue stores the reduction of length of shortest path (after the change of an edge weight) from the virtual source to each vertex instead of the actual length of the shortest path from the virtual source. The reduction of the shortest path to vertex $v$ is denoted $\Delta_{v}$. In Algorithm 2, $Q$ is a min-heap based queue and contains priorityvertex pairs. $h$ is the outdated VSDV and $w_{f g}$ is a decremented edge weight. The algorithm is illustrated in (e-g) of Figure 4.

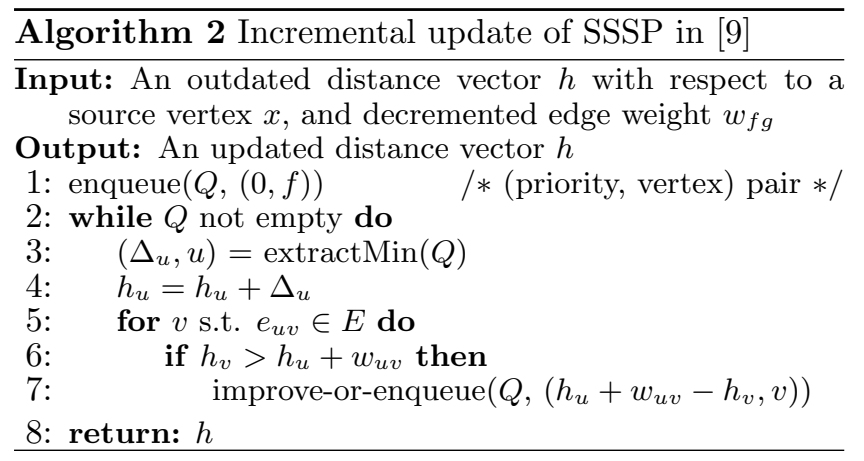

Let $V_{H} \subseteq V$ and $E_{H} \subseteq E$ be the vertices and edges visited by the incremental update algorithm, which are shown red in Figure 4(f). The complexity is $O\left(V_{H} \log \right.$ $\left.V+E_{H}\right)$ because the update is similar to Dijkstra's algorithm.

\subsection{Complexity analysis}

In Table 3 the complexities for the M- and FS-scheduler to perform the different operations are summarized. In the table $V_{H}, V_{F S}, V_{F S K}, E_{H}, E_{F S}$, and $E_{F S K}$ are subsets of $V$ and $E$, respectively, and $2 \leq K \leq 2 k$. From the table, the M-scheduler and FS-scheduler seem to be suitable for different applications. The M-scheduler can perform compute-FSR and compute-NN-FSRs operations efficiently, whereas the FS-scheduler can perform update-SCG operations faster.

Table 3: Overview of the scheduler complexities.

\begin{tabular}{|c|c|c|}
\hline Operation & \multicolumn{2}{|c|}{ Scheduler } \\
\hline & M & FS \\
& in $[1]$ & in this chapter \\
\hline Initialization & $O\left(V^{3}\right)$ & $O(V E)$ \\
Compute-FSR & $O(1)$ & $O\left(V_{F S} \log V+E_{F S}\right)$ \\
Compute-NN-FSRs & $O(k)$ & $O\left(K\left(V_{F S K} \log V+E_{F S K}\right)\right)$ \\
Update-SCG & $O\left(V^{2}\right)$ & $O\left(V_{H} \log V+E_{H}\right)$ \\
\hline Memory & $O\left(V^{2}\right)$ & $O(V)$ \\
\hline
\end{tabular}

\subsection{Experimental results on clock schedul- ing}

Our proposed schedulers are implemented in $\mathrm{C}++$ and the experiments are performed on a quad core 3.10 $\mathrm{GHz}$ Linux machine with $7.7 \mathrm{GiB}$ of memory. 
Table 4: Properties of the ISCAS89 and the Derived benchmark suite.

\begin{tabular}{|c|r|r|}
\hline Name & $\begin{array}{c}\text { Sinks } \\
\text { (num) }\end{array}$ & $\begin{array}{c}\text { Edges } \\
\text { (num) }\end{array}$ \\
\hline s1423 & 74 & 78 \\
s5378 & 179 & 175 \\
s15850 & 597 & 318 \\
\hline
\end{tabular}

Three sets of benchmarks are used to evaluate the proposed schedulers, the ISCAS89 [10] benchmark suite, a derived (D) benchmark suite based on a 45-nm technology [4], and a synthesized Open Cores (OC) benchmark suite. The ISCAS89 benchmarks are used to compare our work with the previous work of [1]. We acknowledge that these ISCAS89 benchmarks are small (see Table 4). Therefore, we derive a set of benchmarks with predefined number of sequential elements to stresstest our schedulers (see Table 4). These benchmarks are constructed with parameters from a $45 \mathrm{~nm}$ technology [4]. Finally, we synthesize a set of Open Cores verilog specifications [11] with Synopsys DC and Synopsys ICC, using a $32 \mathrm{~nm}$ library, to obtain 5 benchmarks with more realistic placement and timing constraints (see Table 5). The derived and Open Cores benchmarks are available online [12].

Table 5: Summary of the Open Cores benchmarks.

\begin{tabular}{|c|r|r|r|}
\hline Name & $\begin{array}{c}\text { Clock } \\
\text { period } \\
\text { (ns) }\end{array}$ & $\begin{array}{c}\text { Number of } \\
\text { sequential } \\
\text { elements }\end{array}$ & $\begin{array}{c}\text { Number } \\
\text { of skew } \\
\text { constraints }\end{array}$ \\
\hline msp & 12.3 & 683 & 44990 \\
fpu & 40.0 & 715 & 16263 \\
ecg & 1.0 & 7674 & 63440 \\
aes & 1.0 & 13216 & 53382 \\
jpeg & 1.5 & 57491 & 496727 \\
\hline
\end{tabular}

\subsubsection{Comparison with [1] on ISCAS89}

In Table 6, we compare the performance of our algorithms with the results reported in [1] in terms of wire length and run-time on the ISCAS89 benchmark suite. In the table, the wire length results in [1] are normalized with our wire length results. As we have mimicked their implementation, we expect very similar results in terms of wire length, as shown in the table. Now that we have established that our own implementation has similar performance, we will turn our focus on the runtime. We re-implemented the M-scheduler as the results in [1] are obtained on an older machine. We show both the run-time of M-scheduler as reported in [1] and the run-time of our implementation of M-scheduler in Table 6 . In the remainder, we show the run-time of our implementation of M-scheduler.

\subsubsection{Results on the Derived and Open Cores benchmark suits}

In Table 7 and Figure 5 the run-time results of the
Table 6: Comparison of run-time and wire length with [1] on the ISCAS89 benchmark. In the table the wire length results in [1] are normalized with our results. The reported runtimes are for the Greedy-UST/DME algorithm.

\begin{tabular}{|c|r|r|r|r|}
\hline & \multicolumn{3}{|c|}{ Run-time in (s) } & \multicolumn{2}{c|}{$\begin{array}{c}\text { Normalized } \\
\text { wire length }\end{array}$} \\
\hline & M & M & \multicolumn{1}{|c|}{ FS } & \multicolumn{1}{|c|}{ Greedy- } \\
\cline { 3 - 5 } & {$[1]$} & \multicolumn{2}{|c|}{ in this work } & UST/DME \\
\hline s1423 & 1 & 1 & 1 & 1.00 \\
s5378 & 8 & 1 & 1 & 1.01 \\
s15850 & 288 & 4 & 1 & 0.98 \\
\hline
\end{tabular}

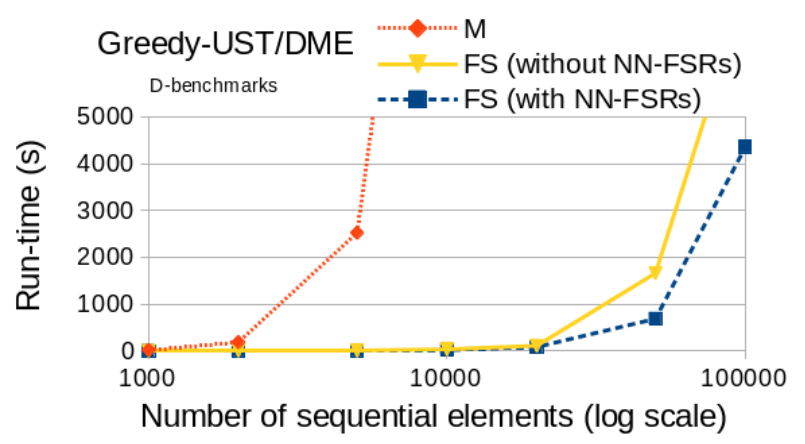

Figure 5: Run-time of Greedy-UST/DME algorithm.

Greedy-UST/DME algorithm are reported and illustrated, respectively. In the table, both the total runtime and the run-time consumed by the clock scheduler are listed. For the FS-scheduler, we report the run-time both with and without the NN-FSRs operation enabled.

From the table and the figure, we find that the FSscheduler seems to be more scalable compared with the M-scheduler. The FS-scheduler with the NN-FSRs operation enabled is more than twice as fast as without the NN-FSRs operation enables. The run-time differences stem from that multiple requested shortest paths can be computed at the same time, avoiding redundant computations. Nevertheless, even with the FS-scheduler, the scheduler is consuming a majority of the run-time of the Greedy-UST/DME application. Based on the run-time complexities, the M- and FS-scheduler will be advantageous for different applications, depending on the ratio of Compute-FSR and Update-SCG calls. Clearly from the experimental results the Greedy-UST/DME application falls into the category of applications where the FS-scheduler seems to be more advantageous. We believe the main reason for that is that it is costly to maintain all the shortest paths of the SCG, when relatively few paths are queried using compute-FSR and compute-NN-FSRs operations.

\section{THE PROPOSED UST-LTSM FRAME- WORK}

The flow of the proposed UST-LTSM framework is 
Table 7: Greedy-UST/DME run-times. $t_{t o t}$ denotes the total run-time and $t_{s}$ denotes the runtime of the scheduler. A ' $-(t)$ ' or ' $-(m)$ ' means that the run-time is more than 15 hours, and the memory is insufficient, respectively.

\begin{tabular}{|c|c|c|c|c|c|c|}
\hline \multirow[t]{3}{*}{ Circuit } & \multirow{2}{*}{\multicolumn{2}{|c|}{$\mathrm{M}$}} & \multicolumn{4}{|c|}{ FS } \\
\hline & & & \multicolumn{2}{|c|}{ without NN-FSRs } & \multicolumn{2}{|c|}{ with NN-FSRs } \\
\hline & $\begin{array}{c}t_{\text {tot }} \\
(\mathrm{s})\end{array}$ & $\begin{array}{c}t_{s} \\
(\mathrm{~s})\end{array}$ & $\begin{array}{c}t_{t o t} \\
(\mathrm{~s})\end{array}$ & $\begin{array}{l}t_{s} \\
(\mathrm{~s})\end{array}$ & $\begin{array}{c}t_{t o t} \\
(\mathrm{~s})\end{array}$ & $\begin{array}{l}t_{s} \\
(\mathrm{~s})\end{array}$ \\
\hline D-1 & 18 & 17 & 2 & 2 & 1 & 1 \\
\hline D-2 & 188 & 187 & 7 & 7 & 5 & 5 \\
\hline D-3 & 2524 & 2519 & 9 & 6 & 5 & 3 \\
\hline D-4 & 16725 & 16698 & 44 & 34 & 17 & 13 \\
\hline D-5 & $-(\mathrm{T})$ & $-(\mathrm{T})$ & 164 & 111 & 85 & 62 \\
\hline D-6 & $-(\mathrm{M})$ & $-(\mathrm{M})$ & 2112 & 1662 & 687 & 468 \\
\hline D-7 & $-(\mathrm{M})$ & $-(\mathrm{M})$ & 9534 & 7706 & 4359 & 3493 \\
\hline msp & 4 & 4 & 3 & 2 & 1 & 1 \\
\hline fpu & 6 & 5 & 3 & 2 & 2 & 1 \\
\hline ecg & 8105 & 7902 & 121 & 112 & 68 & 63 \\
\hline aes & 38037 & 37979 & 657 & 63 & 358 & 343 \\
\hline jpeg & $-(\mathrm{M})$ & $-(\mathrm{M})$ & 8985 & 8364 & 4262 & 3956 \\
\hline
\end{tabular}

shown in Figure 6 and illustrated with an example in Figure 7. The UST-LTSM framework takes an input design with skew constraints and a user specified safety margin $M_{u s e r}$, and constructs a useful skew clock tree with large tailored safety margins.

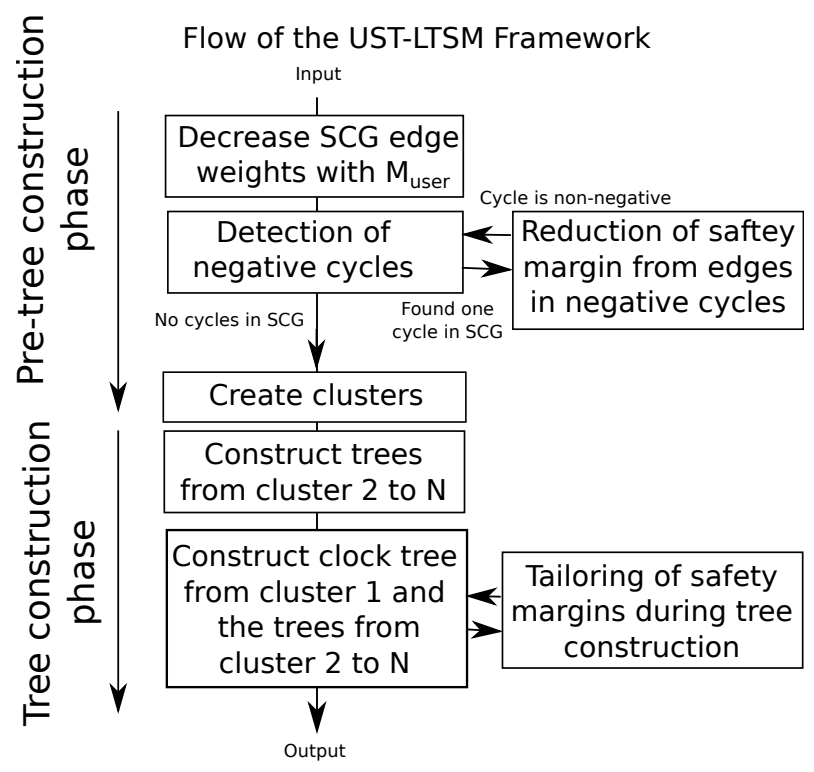

Figure 6: The flow for the proposed UST-LTSM framework.

A user specified safety margin $M_{\text {user }}$ is introduced to all the skew constraints in the SCG constructed from Eq. (3) (note that this is the set of constraints without safety margins). Inserting a safety margin is equivalent to decreasing the weight of the corresponding edge in the SCG. In the example in Figure 7, a safety margin $M_{\text {user }}=2$ is inserted to the skew constraints in the SCG in (a) to form the updated SCG in (b). Note that the original SCG in Figure 7(a) has a maximum uniform safety margin $M=1$. All cycles with an average margin of less than $M_{\text {user }}$ will now become negative. As

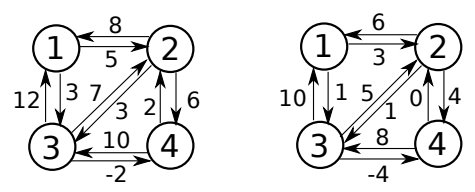

(a) SCG

(b) SCG with a Muser inserted
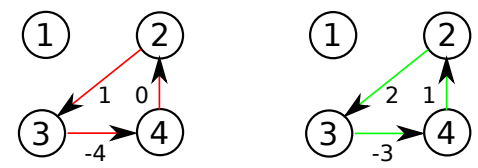

$\mathrm{C}_{1} \mathrm{C}_{2}$ $1 \longdiv { 2 3 4 }$

$\begin{array}{llll}\text { (c) Negative cycle } & \text { (d) Reduction of safety margin } & \text { (e) Create clusters }\end{array}$

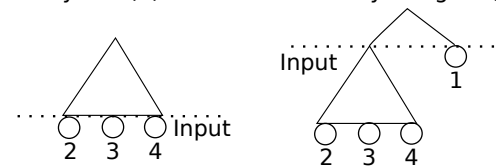

(f) Tree from $C_{2}$ (g) Tree from sinks of $C_{1}$ and tree of $C_{2}$

Figure 7: Illustration of the proposed USTLTSM framework.

$M_{\text {user }}>M$, at least one negative cycle will be created, i.e., no feasible clock schedule exists.

To recover a feasible clock schedule, all the created negative cycles must be removed by increasing the weights of the edges in the cycle, i.e., reducing the safety margins. A feasible clock schedule is recovered when we reduce the safety margin of each edge $e_{i j}$ to its respective maximum margin $M_{i j}^{\max }$, as defined in Section 3.3. In Figure 7(c), a negative cycle is illustrated and after the safety margins on the edges have been reduced, the non-negative cycle in Figure $7(\mathrm{~d})$ is obtained. The negative cycle detection is detailed in Section 6.1 and reduction of safety margin is explained in Section 6.2.

After all the negative cycles have been removed, all the loose edges have a safety margin of $M_{\text {user }}$ and all the tight edges have safety margins less than $M_{\text {user }}$, but at least $M$. As the safety margins have been reduced for tight edges, the corresponding sinks to these skew constraints should be placed close in the topology to lower the point of divergence. We join such pairs of sinks before we construct the remainder of the clock tree to ensure a low point of divergence for these sinks in the tree topology. To facilitate that, we cluster all sinks in a cycle of tight edges together. If two clusters share some common sinks, the two clusters are grouped together to form a larger cluster. (There are other ways of clustering sinks. We choose to group clusters together because only a limited number of clusters are found for the circuits we have considered. Moreover, each cluster contains only a few sinks.) All other sinks not in any negative cycles are grouped together to form another cluster $C_{1}$.

In Figure 7(e), we show two clusters: $C_{2}$ is formed by the three sinks in the cycle of tight edges, and $C_{1}$ contains a singleton sink. In reality, we expect $\left|C_{1}\right|$ to be much larger.

Assume that $N$ sink clusters $C_{1}, \ldots, C_{N}$ have been 
formed. Next, $N-1$ clock trees are constructed, one for each of the clusters $C_{2}, \ldots, C_{N}$, using the sinks of the respective clusters, which is shown in Figure 7 (f). Note that the constructed clock trees may contain buffers (as shown in Figure 7(f)). Next, a clock tree is constructed using the sinks of cluster $C_{1}$ and the $N-1$ trees obtained for clusters $C_{2}, \ldots, C_{N}$, which is shown in Figure $7(\mathrm{~g})$.Furthermore, while constructing the clock trees, for the respective clusters, the inserted safety margins are tailored based on topological commitments. The details of the construction of the buffered USTs with tailored safety margins is provided in Section 6.3.

A limitation of this work is that for designs with extremely uniform and regular skew constraints, such an approach would not be particularly advantageous. For such designs, there is only one cluster, and the GreedyUST/DME approach [1] would suffice. However, in the synthesized designs from [11], the slacks in the timing constraints are rather non-uniform. The details of the synthesized designs are provided in Table 5.

\subsection{Detection of Negative Cycles}

The negative cycle detection (NCD) problem is that of determining if an SCG contains a negative cycle and if any such negative cycles exists, find one such cycle. The general NCD problem has been studied extensively and an excellent review of several efficient algorithms using different auxiliary data structures is provided in [13].

We use a practical approach based on the BellmanFord algorithm [6] to solve the NCD problem with a worst case run-time complexity of $O(V E)$. However, our algorithm performs much better than $O(V E)$ on the average because it contains two early termination features.

When no negative cycles exist, the Bellman-Ford algorithm can terminate early if no shortest paths are updated in successive iterations [6]. It is also known that if a graph contains a negative cycle, there is a chance that the cycle can be detected before $V$ iterations of edge relaxations, by tracing predecessors from a vertex $v$, especially when the cycle size is small [13]. Therefore, the algorithm can again terminate early. The two early termination methods are incorporated into the Bellman-Ford algorithm.

\subsection{Reduction of safety margin from edges in negative cycles}

A negative cycle in the SCG indicates that no feasible clock schedule exists, i.e., some or all the edges in the cycle have been allocated infeasible safety margins higher than the maximum margin of each respective edge. Note that this situation was intentionally created by inserting safety margin $M_{\text {user }}$ on all the edges. To remove the negative cycle, safety margin equal to the magnitude of the total weight of the negative cycle must be reduced from the edges of the cycle. Two techniques can be considered to distribute the reduction of the safety margins across the edges of the cycle:
- Reduce the safety margin uniformly from all edges of the negative cycle.

- Reduce the safety margin non-uniformly from the edges in the negative cycle.

The first approach maximizes the minimum safety margin in the skew constraints of the cycle. Using the first method it is straightforward to reduce the amount of safety margin on the edges of the cycle. By definition, we can guarantee that all edges will have a safety margin $m$, with $M \leq m<M_{u s e r}$. The second method reduces the safety margin from fewer edges, which may result in fewer tight edges and smaller clusters.

For the synthesized circuits in [11], the cycles typically contain only a few edges. Therefore, we used only the first approach. However, for circuits with large cycles, we believe that the second approach could be more flexible and effective.

\subsection{Construction of buffered USTs with tai- lored safety margins}

In this section, we give an outline how we construct our buffered useful-skew trees with tailored safety margins. An outline of the flow for the tree construction is given in Figure 8. Our clock trees are constructed buffer stage by buffer stage. The merging process of the Greedy-UST/DME algorithm together with the stem wire and buffer insertion in $[14,15]$ form the core of our proposed flow for the construction of one buffered stage. The core flow is extended two optional techniques of tailoring the safety margins to the topology. This section is focused on these optional tailoring techniques. First, we provide a brief overview of the core of the flow.

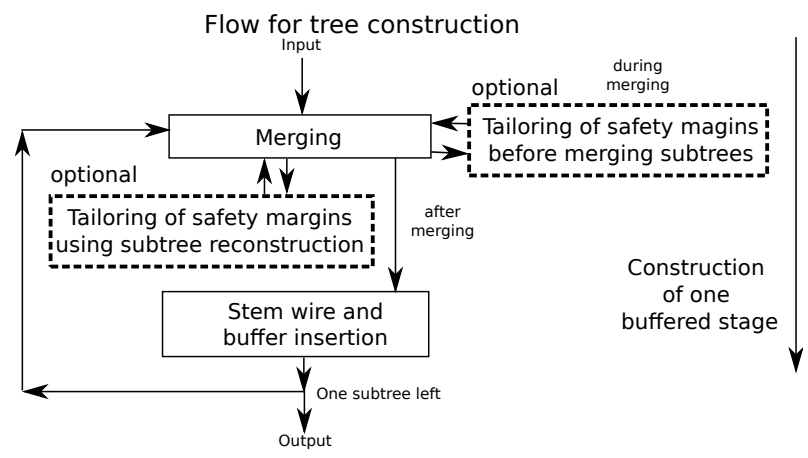

Figure 8: The flow for the construction of buffered USTs with tailored safety margins.

A buffer stage consists of a number of subtrees each driven by a buffer. The input to the construction of a buffer stage is either the clock sinks or the buffers driving the subtrees of the downstream buffer stage. In the merging process, larger subtrees are formed by iteratively pairwise merging smaller subtrees together. The selection of the subtrees that are to be merged is done using a NNG where the distance between two subtrees is defined to be the wiring cost of merging that pair 
of subtrees. The wiring cost of merging two subtrees is computed based on the spatial distance, the timing, and the FSR of the pair. If no detour wiring is required, the wiring cost is equal to the Manhattan distance between the subtrees, otherwise the wiring cost is equal to the Manhattan distance plus the length of the detour wiring [1]. (Detour wiring is needed when the relative timing of the subtrees is required to be adjusted to meet the skew constraints captured by the FSR.) The length of the required detour wiring is computed analytically using the Elmore delay model as in [1]. However, it is time consuming to compute the distance of each subtree-pair. Therefore, only the distance between subtree-pairs that are spatially close (or neighbors) are computed, as the distances between these subtree-pairs are expected to be small. These neighboring subtree pairs are determined based on Delaunay triangulation [16]. Given a Delaunay triangulation, we define two subtrees to be neighbors if they are separated by three or fewer edges in the triangulation. (An equally good alternative is to use the partitioning method in [1].) To compute the distances between the neighboring subtree-pairs, the required FSRs are computed using the Compute-NNFSRs operation. Next, the subtree-pairs that are closest in the NNG are selected to be merged.

The merging process of the selected subtree pairs is guided by the compute-FSR and update-SCG operations as detailed in Algorithm 1 in Section 3.2.

After two subtrees $T_{u}$ and $T_{w}$ have been merged to form a single subtree $T_{v}$, the $10 \%$ to $90 \%$ transition time, is evaluated. If the transition time of $T_{v}$ is greater than a transition time constraint $S_{\text {tran }}$, the two subtrees $T_{u}$ and $T_{w}$ are unmerged and locked from further merging. After all the subtrees have been locked, a piece of stem wire is extended from the root of every locked subtree (as in [14, 15]). Next, a buffer is inserted to drive each subtree through the inserted stem wire. Iteratively, the merging in the NNG and buffer insertion are performed until all subtrees have been merged to a single tree.

Now, we present the proposed optional techniques of tailoring the safety margins to the tree topology. Our tailoring of the safety margins is based on incrementally reducing the safety margin that was inserted in the pre-tree construction phase. The main advantage of the proposed approach compared with the incremental insertion of safety margins, in [2], is that we cannot run out of safety margins. The first tailoring method tailors safety margin when two subtrees are merged (similar to in [2]). The second method is based on reconstructing a subtree in order to tailor the safety margins in many skew constraints, at the same time. However, we do not apply the two optional tailoring methods simultaneously.

\subsubsection{Tailoring of safety margins prior to merg- ing subtrees}

This technique is applied when two subtrees are merged
(In line 6 of Algorithm 1 in Section 3.2). The maximum negative degradation that can be introduced by variations in skew constraints between sinks in the two subtrees is estimated. The estimation is performed by computing the maximum root-to-sink delay of the subtrees that are about to be merged. Next, the degradation is determined as the sum of the maximum root-to-sink delay of the two subtrees multiplied by a factor $p$. (The factor $p$ is set to 0.075 based on an empirical study to trade off cost and robustness.) Next, the safety margin on the edges in the SCG between sinks residing in the two respective subtrees are set to the minimum of the currently inserted safety margin and the estimated required safety margin.

Reducing the safety margin corresponds to increasing edge weights in the SCG (see Eq. (4)), which inturn elongates the shortest paths that define the FSRs, resulting in expanded FSRs and reduced wiring costs.

\subsubsection{Tailoring of safety margins using subtree reconstruction}

The reconstruction technique is applied to each of the subtrees formed in the merging step. An outline of the reconstruction of a single subtree is provided in Algorithm 3.

The reconstruction step is only performed on the part of a subtree that was constructed during the last merging process, i.e., subtree constructed above the last inserted buffers or the clock sinks, both referred to as stage sinks. The reconstruction is performed by collecting the stage sinks of the subtree that is about to be reconstructed. Next, the maximum safety margin that is required in the skew constraints between the collected stage sinks is estimated; we refer to this safety margin as $M_{\text {est }}$. The estimation is performed similarly as in the previous section, the difference is that the estimate of the safety margin is based on the root-to-sink delay of the subtree that is about to be reconstructed. Next, the safety margin in each of the skew constraints spanning between the sinks of the subtree is set to the minimum of the estimate of the required safety margin $M_{e s t}$ and the currently inserted safety margin. Lastly, the collected stage sinks of the subtree are inserted into an NNG and the subtree is reconstructed.

After the reconstruction the wiring cost of the initial subtree is compared with the reconstructed subtree in terms of wiring cost, i.e., wire length. The subtree of least cost is kept. If the initial subtree is kept, the SCG is restored to the state before the reconstruction was applied. Naturally, if the sinks of the subtree cannot be reconstructed to a subtree without violating the transition time constraint, the initial subtree is kept.

After all the subtrees have been reconstructed, we apply the merging process again to the reconstructed subtrees. As some subtrees may have been reconstructed with smaller wiring cost, it may be possible to perform additional merges, reducing the number of subtrees that are required to be buffered. Based on a run- 
time performance trade-off, we apply the subtree reconstruction only once in the construction of each stage (although it is possible to apply the reconstruction iteratively). We also note that without the use of the proposed FS-scheduler, the run-time of the reconstruction step would have been prohibitively long, as the reconstruction of each subtree requires additional FSRs to be computed.

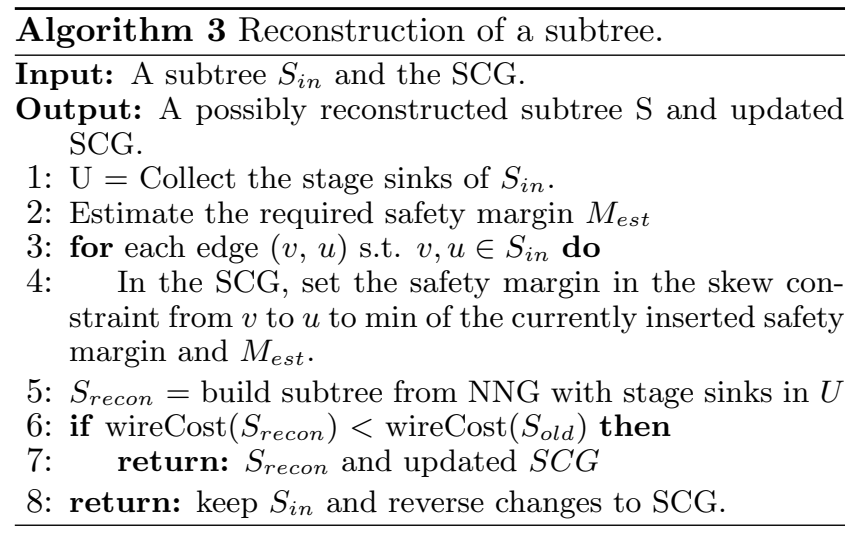

A limitation of the LTSM framework is that the size of a buffered UST constructed from a cluster with a large number of sinks may be large, i.e., the point of divergence for certain sink-pairs residing in the same cluster may be high. As a result, timing violations may occur in timing constraints where an adequate safety margin is not inserted. However, on the considered circuits, there are only few sinks in the clusters where the full safety margin $M_{u s e r}$ is not provided, i.e., the clusters $C_{2}$ to $C_{N}$.

\section{EVALUATION OF THE UST-LTSM FRAMEWORK}

\subsection{Experimental setup}

The same machine that was used for the scheduling experiments is used to obtain the results in this section. We use a scaled version of the ISCAS89 benchmark suite and the Open Cores benchmark suite in our evaluation (see Table 4 and Table 5). The ISCAS89 benchmark suite is scaled because the skew constraints in that suite are too loose to be representative of today's designs with tight skew constraints. The scaled_ISCAS benchmarks are obtained by scaling the ISCAS89 circuits in terms of physical locations of the sinks, the load capacitances, propagation delays, and operating frequency based on the ITRS road-map. The benchmarks are available in an online repository [12].

To evaluate the synthesized USTs robustness to variations, an adoption of the Monte Carlo simulation framework in the International Symposium on Physical Design (ISPD) 2010 contest [4] is used. In the Monte Carlo simulation framework, constructed clock trees are affected by wire width variations $( \pm 5 \%)$ and supply voltage variations $( \pm 7.5 \%)$ around the respective nominal values, as in the ISPD 2010 contest. In addition, we extend the variations model to also include temperature variations $( \pm 15 \%)$, and channel length variations $( \pm 5 \%)$ around the nominal respective values. Moreover, all the variations are assumed to exhibit spatial correlation using a 5-level quad-tree model [17]. The variations are evenly assigned to the bottom three levels of the quad-tree. The variations in each level of the quadtree are generated using a uniform distribution. This is an improvement over the ISPD 2010 contest variations model where the variations exhibited no spatial correlation. It is also an improvement over the variations model in [15] where devices placed at the same location experienced the same voltage variations. We assume the same technology parameters for the devices and wires in the ISPD 2010 contest [4]. Timing evaluation of the constructed clock trees is performed using a stage-bystage SPICE simulation approach using transition time propagation [18].

The skew performance of each clock network is evaluated using its yield and timing slack. Given the actual timing obtained through simulation, we define the slack in the setup and hold time constraints in Eq. (3) to be $u_{i j}-s k e w_{i j}$ and $s k e w_{i j}-l_{i j}$, respectively. Positive slacks imply that the constraints are satisfied while negative slacks correspond to constraint violations. The transition time constraint $S_{\text {tran }}$ is set to $100 \mathrm{ps}$.

The yield is measured by simulating each clock network with 500 Monte Carlo simulations, with variations applied as specified earlier. Each Monte Carlo simulation represents a testing of a chip. If all the skew constraints and the transition time constraints are satisfied, we classify the tested chip as a good chip. The yield is obtained by dividing the number of good chips by the total number of chips tested. The $95 \%$-slack is obtained by recording the worst slack in each Monte Carlo simulation. The $95 \%$-slack is defined to be the 95\%-tile of the 500 worst slacks (ordered in descending order). We also report the capacitive cost as this correlates with the power consumption of the clock network. The run-times of the synthesis tool are given in the columns labeled "Run-time" in the tables.

We introduce the following acronyms for the different structures proposed in this work and in $[1,2,14]$. The approach of constructing a clock tree with uniform safety margins in [1], is denoted USM. The method of narrowing the FSRs to provide safety margins in [2] is denoted NSM. (Note that this is our reimplementation of the work in [2], as the original source code is not available.) We also construct a buffered zero skew tree (ZST), using a method similar to that in [14]. Next, we describe the structures constructed using the UST-LTSM framework. The baseline structure constructed by the framework is the large safety margin (LSM) structure and it is constructed using only negative cycle detection and clustering. By deactivating the clustering (no clustering) but inserting the same safety margins the NC-LSM structure is obtained. P-LSM is 
Table 8: Performance of the USTs constructed for benchmark circuits with loose skew constraints.

\begin{tabular}{|c|c|c|c|c|c|c|}
\hline $\mathrm{BM}$ & Name & $\begin{array}{l}\text { Safety } \\
\text { margin } \\
(\text { ps })\end{array}$ & $\begin{array}{l}\text { Yield } \\
(\%)\end{array}$ & $\begin{array}{l}95 \%- \\
\text { slack } \\
(\mathrm{ps})\end{array}$ & $\begin{array}{l}\text { Cap } \\
(\mathrm{pF})\end{array}$ & $\begin{array}{c}\text { Time } \\
\text { (s) }\end{array}$ \\
\hline scaled & $\mathrm{ZST}$ & $\mathrm{n} / \mathrm{a}$ & 100 & 140.0 & 4.59 & 44 \\
\hline \multirow[t]{5}{*}{ _s1423 } & NSM & $\mathrm{n} / \mathrm{a}$ & 100 & 140.0 & 3.41 & 74 \\
\hline & LSM/USM & 0 & 100 & 140.0 & 3.41 & 17 \\
\hline & LSM/USM & 50 & 100 & 140.0 & 3.41 & 19 \\
\hline & P-LSM & 50 & 100 & 140.0 & 3.41 & 19 \\
\hline & R-LSM & 50 & 100 & 140.0 & 3.53 & 55 \\
\hline scaled & $\mathrm{ZST}$ & $\mathrm{n} / \mathrm{a}$ & 100 & 12.9 & 6.78 & 76 \\
\hline \multirow[t]{5}{*}{ _s5378 } & NSM & $\mathrm{n} / \mathrm{a}$ & 100 & 12.9 & 5.71 & 116 \\
\hline & LSM/USM & 0 & 100 & 12.9 & 5.71 & 33 \\
\hline & LSM/USM & 50 & 100 & 38.9 & 8.52 & 72 \\
\hline & P-LSM & 50 & 100 & 25.0 & 8.04 & 112 \\
\hline & R-LSM & 50 & 100 & 37.7 & 7.99 & 433 \\
\hline \multirow[t]{6}{*}{$\mathrm{msp}$} & $\mathrm{ZST}$ & $\mathrm{n} / \mathrm{a}$ & 100 & 80.0 & 2.32 & 27 \\
\hline & NSM & $\mathrm{n} / \mathrm{a}$ & 100 & 80.0 & 1.81 & 60 \\
\hline & LSM/USM & 0 & 100 & 80.0 & 1.81 & 21 \\
\hline & LSM/USM & 50 & 100 & 80.0 & 1.81 & 26 \\
\hline & P-LSM & 50 & 100 & 80.0 & 1.81 & 58 \\
\hline & R-LSM & 50 & 100 & 80.0 & 1.76 & 56 \\
\hline \multirow[t]{6}{*}{ fpu } & $\mathrm{ZST}$ & $\mathrm{n} / \mathrm{a}$ & 100 & 50.0 & 2.90 & 59 \\
\hline & NSM & $\mathrm{n} / \mathrm{a}$ & 100 & 50.0 & 2.07 & 70 \\
\hline & LSM/USM & 0 & 100 & 50.0 & 2.07 & 25 \\
\hline & LSM/USM & 50 & 100 & 50.0 & 2.07 & 26 \\
\hline & P-LSM & 50 & 100 & 50.0 & 2.07 & 67 \\
\hline & R-LSM & 50 & 100 & 50.0 & 2.11 & 60 \\
\hline \multirow[t]{6}{*}{ aes } & $\mathrm{ZST}$ & $\mathrm{n} / \mathrm{a}$ & 0 & -18.9 & 40.91 & 1855 \\
\hline & NSM & $\mathrm{n} / \mathrm{a}$ & 0 & -29.6 & 125.16 & 5011 \\
\hline & LSM/USM & 0 & 0 & -25.3 & 39.99 & 1727 \\
\hline & LSM/USM & 50 & 95 & -0.5 & 368.86 & 10294 \\
\hline & P-LSM & 50 & 96 & 1.3 & 376.44 & 15936 \\
\hline & R-LSM & 50 & 97 & 5.3 & 250.20 & 7396 \\
\hline \multirow[t]{6}{*}{ jpeg } & $\mathrm{ZST}$ & $\mathrm{n} / \mathrm{a}$ & 0 & -31.0 & 150.76 & 43417 \\
\hline & NSM & $\mathrm{n} / \mathrm{a}$ & 0 & -28.2 & 144.86 & 25769 \\
\hline & LSM/USM & 0 & 0 & -24.9 & 129.63 & 25793 \\
\hline & LSM/USM & 50 & 92 & -4.9 & 377.44 & 44599 \\
\hline & P-LSM & 50 & 89 & -14.8 & 385.14 & 183107 \\
\hline & R-LSM & 50 & 93 & -6.8 & 301.46 & 104549 \\
\hline
\end{tabular}

the LSM structure combined with the tailoring of safety margins prior to merging subtrees. R-LSM is the structure obtained when the LSM structure is combined with subtree reconstruction tailoring technique.

We assume that the user specified safety margin $M_{u s e r}$ will never be larger than $M_{u s e r}^{\max }=50$ ps. If a benchmark circuit has $M>M_{u s e r}^{\max }$, we say the circuit has loose skew constraints; otherwise, we say that the benchmark circuit has tight skew constraints. The benchmark circuits scaled_s1423, scaled_s5378, msp, fpu, aes, and jpeg have loose skew constraints and scaled_s15850 and ecg have tight skew constraints. The maximum uniform safety margins for scaled_s15850 and ecg are $M=27$ ps and $M=15 \mathrm{ps}$, respectively.

The experimental results for the benchmark circuits with loose skew constraints are presented in Table 8 . The structures that are compared are USM, NSM, ZST, LSM, P-LSM and R-LSM. The structures USM and LSM are constructed with a safety margins of 0 and 50 ps and the structures P-LSM and R-LSM are constructed with a safety margin of 50 ps. Note that the LSM structure is equivalent to the USM method on benchmarks with loose design constraints and they are therefore denoted LSM/USM.

\subsection{Designs with loose skew constraints}

In Table 8, we observe that all the different methods can achieve a yield of $100 \%$ on the benchmark circuits scaled_s1423, scaled_s5378, msp, and fpu. The capacitive cost on these benchmark circuits is also similar, except that the ZST is significantly more expensive. This demonstrates that the proposed framework can reduce the cost by taking advantage of the non-uniform skew constraints, a main contribution of [1]. Moreover, the two techniques of tailoring the safety margins to the tree topology have minor, or no, impact on the yield or the capacitive cost. This is because the skew constraints are not very restrictive and therefore there is no need to tailor the safety margins to the tree topology.

On benchmark circuit aes and jpeg, the largest two circuits, none of the structure obtain a yield of $100 \%$ and the differences in capacitive cost between certain structures are quite substantial. To facilitate a more detailed comparison, we construct the USM/LSM, PLSM, and R-LSM structures with a user specified safety margin of $0,10,20,30,40$, and 50 . The results are presented in Table 9 and Figure 9 . It is observed that neither the ZST nor the NSM structure can obtain an adequate yield on the circuits aes and jpeg. The ZST cannot obtain high yield because useful skew is required in certain skew constraints. The yield loss of the NSM structure [2] is caused by the lack of safety margin at the top of the tree (confirmed experimentally).

The LSM/USM, P-LSM, R-LSM structures smoothly trade-off yield and capacitive cost by increasing the user specified safety margin. Moreover, the tailoring techniques have minor, or no, impact on the yield. For the same safety margin $M_{u s e r}$, the yield of the LSM/USM, P-LSM, and R-LSM structures are similar. This is also expected because the tailoring techniques are aimed at reducing the capacitive cost without negatively affecting the yield. Furthermore, the technique of adjusting safety margins prior to merging subtrees has a marginal impact on the total capacitive cost. (The capacitive cost of the P-LSM is lower than that of the LSM/USM structure for certain values of the user specified safety margin and higher for other values.) However, the technique of tailoring the safety margins by reconstruction is effective and successfully reduces the capacitive cost substantially. We believe that the superiority of the tailoring in the R-LSM structure over the P-LSM structure is because the tailoring technique based on subtree reconstruction is capable of capturing more information about the topology at once, and can therefore perform the tailoring of the safety margins more effectively. (We have investigated combining the two tailoring techniques together. However, combining the two techniques results in longer run-times and produces clock trees have similar capacitive cost as the R-LSM structures.)

\subsection{Designs with tight skew constraints}

For the benchmarks circuits with tight skew constraints, the UST-LTSM framework is substantially different compared with [1] because negative cycle detection and clustering are applied. The structures we compare are ZST, 
Table 9: Performance of the USTs constructed on aes and jpeg.

\begin{tabular}{|c|c|c|c|c|c|c|}
\hline$\overline{\mathrm{BM}}$ & Name & $\begin{array}{c}\text { Safety } \\
\text { margin } \\
(\mathrm{ps})\end{array}$ & $\begin{array}{c}\text { Yield } \\
(\%)\end{array}$ & $\begin{array}{c}95 \%- \\
\text { slack } \\
(\mathrm{ps})\end{array}$ & $\begin{array}{l}\text { Cap } \\
(\mathrm{pF})\end{array}$ & $\begin{array}{c}\text { Time } \\
\text { (s) }\end{array}$ \\
\hline \multirow[t]{19}{*}{ aes } & $\overline{\mathrm{ZST}}$ & $\mathrm{n} / \mathrm{a}$ & 0 & -18.9 & 40.91 & 1855 \\
\hline & NSM & $\mathrm{n} / \mathrm{a}$ & 0 & -29.6 & 125.16 & 5011 \\
\hline & LSM/USM & 0 & 0 & -25.3 & 39.99 & 1727 \\
\hline & LSM/USM & 10 & 0 & -19.6 & 167.58 & 3846 \\
\hline & LSM/USM & 20 & 15 & -18.5 & 268.72 & 6575 \\
\hline & LSM/USM & 30 & 60 & -17.1 & 312.89 & 7498 \\
\hline & LSM/USM & 40 & 92 & -3.5 & 318.95 & 9305 \\
\hline & LSM/USM & 50 & 95 & -0.5 & 368.86 & 10294 \\
\hline & P-LSM & 10 & 0 & -24.1 & 163.03 & 9911 \\
\hline & P-LSM & 20 & 6 & -19.6 & 264.61 & 12536 \\
\hline & P-LSM & 30 & 71 & -19.1 & 316.30 & 13884 \\
\hline & P-LSM & 40 & 90 & -8.8 & 327.42 & 15656 \\
\hline & P-LSM & 50 & 96 & 1.3 & 376.44 & 15936 \\
\hline & R-LSM & 0 & 0 & -22.3 & 39.07 & 6769 \\
\hline & R-LSM & 10 & 0 & -18.2 & 131.09 & 5920 \\
\hline & R-LSM & 20 & 18 & -16.8 & 190.66 & 7979 \\
\hline & R-LSM & 30 & 77 & -10.8 & 220.48 & 9030 \\
\hline & R-LSM & 40 & 94 & -1.6 & 218.99 & 7396 \\
\hline & R-LSM & 50 & 97 & 5.3 & 250.20 & 10252 \\
\hline \multirow[t]{19}{*}{ jpeg } & $\mathrm{ZST}$ & $\mathrm{n} / \mathrm{a}$ & 0 & -31.0 & 150.76 & 43417 \\
\hline & NSM & $\mathrm{n} / \mathrm{a}$ & 0 & -28.2 & 144.86 & 25769 \\
\hline & LSM/USM & 0 & 0 & -24.9 & 129.63 & 25793 \\
\hline & LSM/USM & 10 & 0 & -23.3 & 182.02 & 29176 \\
\hline & LSM/USM & 20 & 18 & -13.4 & 243.87 & 33017 \\
\hline & LSM/USM & 30 & 62 & -30.5 & 288.19 & 33755 \\
\hline & LSM/USM & 40 & 83 & -17.4 & 331.12 & 38155 \\
\hline & LSM/USM & 50 & 92 & -4.9 & 377.44 & 44599 \\
\hline & P-LSM & 10 & 0 & -21.9 & 176.06 & 85853 \\
\hline & P-LSM & 20 & 18 & -17.6 & 238.62 & 120927 \\
\hline & P-LSM & 30 & 74 & -20.6 & 286.55 & 132193 \\
\hline & P-LSM & 40 & 84 & -19.0 & 325.77 & 153173 \\
\hline & P-LSM & 50 & 89 & -14.8 & 385.14 & 183107 \\
\hline & R-LSM & 0 & 0 & -26.4 & 127.61 & 72258 \\
\hline & R-LSM & 10 & 0 & -22.6 & 169.71 & 81654 \\
\hline & R-LSM & 20 & 14 & -18.8 & 206.84 & 89118 \\
\hline & R-LSM & 30 & 68 & -40.2 & 236.04 & 89628 \\
\hline & R-LSM & 40 & 84 & -15.4 & 256.43 & 97063 \\
\hline & R-LSM & 50 & 93 & -6.8 & 301.46 & 104549 \\
\hline
\end{tabular}

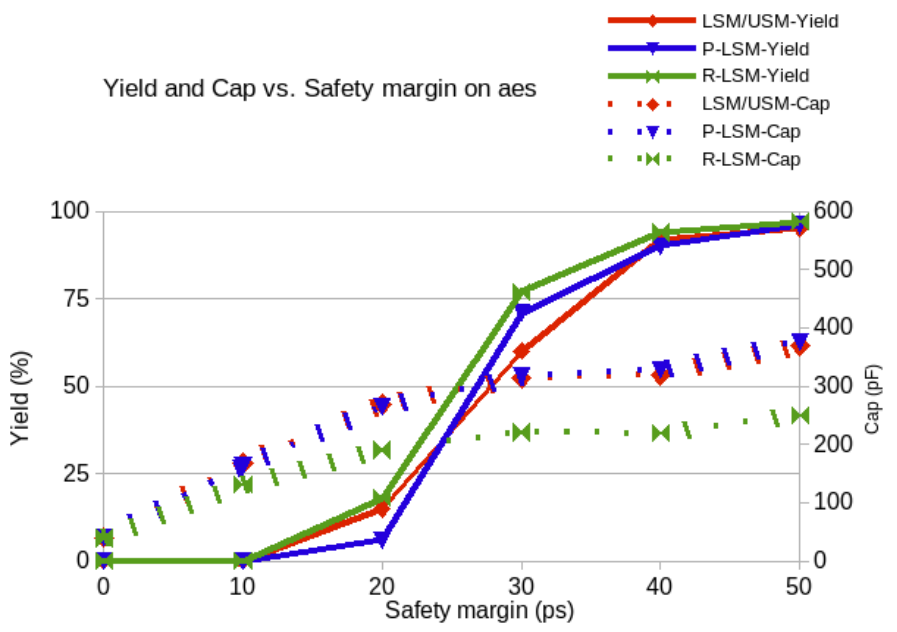

Figure 9: Trade-off between the yield (left vertical axis), capacitive cost (right vertical axis) and the safety margin $M_{u s e r}$ on (horizontal axis) on aes. The yield increases from $0 \%$ to $97 \%$ when $M_{u s e r}$ is increased from 0 ps to 50 ps.
Table 10: Negative cycles found for different settings of $M_{\text {user }}$ on benchmark circuits scaled_s15850 and ecg.

\begin{tabular}{|c|c|r|r|}
\hline BM & $\begin{array}{c}\text { Safety } \\
\text { margin } \\
(\mathrm{ps})\end{array}$ & $\begin{array}{c}\text { Negative } \\
\text { cycles } \\
(\text { num })\end{array}$ & $\begin{array}{c}\text { Early } \\
\text { termination } \\
(\text { num })\end{array}$ \\
\hline scaled_s15850 & $M_{\text {user }}=M+20=47$ & 1 & 1 \\
scaled_s15850 & $M_{\text {user }}=M+40=67$ & 1 & 1 \\
\hline ecg & $M_{\text {user }}=M+15=30$ & 5 & 5 \\
ecg & $M_{\text {user }}=M+20=35$ & 6 & 6 \\
\hline
\end{tabular}

USM, LSM, S-LSM, P-LSM, R-LSM, and R-S-LSM. For the structures where a user specified safety margin is required, various different values are considered, to obtain smooth trade-offs between cost and robustness.

The experimental results on scaled_s15850 and ecg are shown in Table 12. In the table, it can be observed that a user specified safety margin of $M_{\text {user }}=M$ does not obtain a yield of $100 \%$. Therefore, the use of $M_{\text {user }}>M$ is required, which necessitates the use of negative cycles detection and clustering. We focus our attention on the performance of the negative cycle detection and the sink clustering before moving on to the overall performance evaluation of scaled_s15850 and ecg.

\subsubsection{Negative cycle detection and sink cluster- ing}

Negative cycles are found when $M_{\text {user }}>M$. In Table 10, we show the number of negative cycles found in the SCG for different values of $M_{\text {user }}$. We find that our simple early termination procedure is sufficient to perform early termination in every case where a negative cycle exists in the SCG. On benchmark circuit scaled_s15850, there is only one negative cycle when $M_{\text {user }}$ is set to anywhere in the range $M<M_{\text {user }} \leq$ $M+40$ ps. On benchmark circuit ecg, the number of cycles varies for different settings of $M_{u s e r}$, which is shown in Table 10.

The sink clusters constructed based on the negative cycles are shown in Table 11. In addition to the trivial cluster $C_{1}$, one cluster $C_{2}$ is obtained for scaled_s 15850 and on ecg, we obtain two additional clusters when $M_{\text {user }}=M+15 \mathrm{ps}$ and three additional clusters when $M_{\text {user }}=M+20$ ps. On scaled_s15850 the cluster $C_{2}$ contains 3 sinks and on ecg the clusters $C_{2}$ and $C_{3}$ contain a total of seven sinks when $M_{\text {user }}=M+15$ ps. When $M_{\text {user }}=M+20 \mathrm{ps}$, the clusters $C_{2}, C_{3}$, and $C_{4}$ for ecg contain a total of nine sinks. That there are few clusters implies that there are few skew constraints that limit the maximum value of $M$; the UST-LTSM framework was designed specifically to overcome such limitation.

In Table 11, we show the numbers of buffer stages of trees constructed for these clusters in the column labeled "Max stages". A "no" in the column labeled "Clustering?" indicates that the sinks of the "clusters" $C_{2}$ to $C_{K}$ are not joined into separate subtrees before being 
joined with the sinks in the cluster $C_{1}$. It is expected that the "Max stages" of sinks in these "clusters" will be at least as high as those when a subtree is constructed for each of these clusters before joining cluster $C_{1}$. We observe that when clustering is applied, the maximum numbers of buffer stages of the clusters are reduced from 11 or 9 , to 3 on scaled_s15850, and from 6 and 9 , to 3 on ecg. The smaller the buffer stages, the closer are the sinks of the negative cycles in the clock trees. With the ensured lower point of divergence, variations will not affect these sinks too adversely. Moreover, buffer stages above these clusters would have the full safety margin of $M_{\text {user }}$. When the clustering is not applied, the construction of the tree topology is not controlled. Therefore, the "Max stages" on scaled_s15850 happens to be higher for $M_{\text {user }}=47 \mathrm{ps}$ than for $M_{\text {user }}=67 \mathrm{ps \text {, }}$ which may seem counter intuitive.

Table 11: Clusters other than $C_{1}$ created for scaled_s15850 and ecg. The number of such clusters is dependent on $M_{u s e r}$. The "Max stages" columns refer to the numbers of buffer stages of the trees constructed for the clusters.

\begin{tabular}{|c|c|c|c|c|c|}
\hline \multirow[t]{2}{*}{ 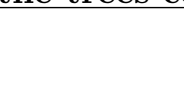 } & \multirow{2}{*}{$\begin{array}{c}\text { Safety } \\
\text { margin } M_{u s e r} \\
(\mathrm{ps})\end{array}$} & \multirow{2}{*}{$\begin{array}{l}\text { Clust- } \\
\text { ering? }\end{array}$} & \multicolumn{3}{|c|}{ Max stages } \\
\hline & & & $\begin{array}{c}C_{2} \\
\text { (num }\end{array}$ & $\begin{array}{c}C_{3} \\
\text { (num) }\end{array}$ & $\begin{array}{c}C_{4} \\
\text { (num) }\end{array}$ \\
\hline scaled_s15850 & $M+20=47$ & yes & 3 & - & - \\
\hline scaled_s15850 & $M+20=47$ & no & 11 & - & - \\
\hline scaled_s15850 & $M+40=67$ & yes & 3 & - & - \\
\hline scaled_s15850 & $M+40=67$ & no & s & - & - \\
\hline ecg & $M+15=30$ & yes & 3 & 1 & - \\
\hline ecg & $M+15=30$ & no & ( & 1 & - \\
\hline ecg & $M+20=35$ & yes & 3 & 1 & 1 \\
\hline ecg & $M+20=35$ & no & s & 1 & 1 \\
\hline
\end{tabular}

\subsubsection{Yield and capacitive cost performance}

In Table 12, it can be observed that the ZST structure, the NSM structure, and the USM structure are not able to obtain adequate yield. (Recall that the USM structure cannot be constructed with a safety margin $M_{\text {user }}$ larger than $M$.) In general, the NC-LSM, LSM, P-LSM, and R-LSM structures, smoothly trade-off yield and capacitive cost by increasing the user specified safety margin. However, the clear yield improvement seems to saturate around $95 \%$ for ecg.

Now we focus on the impact of sink clustering, i.e., the difference between the LSM and the NC-LSM structure (the same safety margins are inserted). When the clustering is deactivated and $M_{\text {user }}$ is increased from 37 ps to $47 \mathrm{ps}$ and $57 \mathrm{ps}$ for scaled_s15850, the yield decreases from $100 \%$ to $98 \%$ and then increases again to $100 \%$. We examine these results in detail and find that for $M_{\text {user }}=47$ the sinks connected with a skew constraint without the full safety margin $M_{\text {user }}$ happen to be placed distant in the tree topology, which results in the yield loss. On the other-hand, in the LSM structure, these sinks are clustered and no yield loss is suffered.

The performance of the tailoring techniques are sim- ilar to the analysis on aes, i.e., the performance of the P-LSM structure is similar to that of the LSM structure in terms of both yield and capacitive cost and the RLSM structure has similar yield as the LSM structure, but the capacitive cost is substantially lower.

Table 12: Experimental results for scaled_s15850 and ecg. On these two benchmarks a $M_{u s e r}>M$ is required to maximize the yield.

\begin{tabular}{|c|c|c|c|c|c|c|}
\hline$\overline{\mathrm{BM}}$ & Name & $\begin{array}{l}\text { Safety } \\
\text { margin } \\
(\mathrm{ps})\end{array}$ & $\begin{array}{l}\text { Yield } \\
(\%)\end{array}$ & $\begin{array}{l}95 \%- \\
\text { slack } \\
\text { (ps) }\end{array}$ & $\begin{array}{l}\text { Cap } \\
(\mathrm{pF})\end{array}$ & $\begin{array}{c}\text { Time } \\
(\mathrm{s})\end{array}$ \\
\hline \multirow{23}{*}{$\begin{array}{c}\text { scaled } \\
\text { _s15850 }\end{array}$} & ZST & $\mathrm{n} / \mathrm{a}$ & 0.0 & -8.5 & 16.9 & 212 \\
\hline & NSM & $\mathrm{n} / \mathrm{a}$ & 82.0 & -4.3 & 14.8 & 139 \\
\hline & LSM/USM & 0 & 22.8 & -16.1 & 14.5 & 231 \\
\hline & LSM/USM & $M=27$ & 99.6 & 9.0 & 20.5 & 383 \\
\hline & NC-LSM & $\mathrm{M}+10=37$ & 100.0 & 14.0 & 25.6 & 1405 \\
\hline & NC-LSM & $M+20=47$ & 98.6 & 7.2 & 32.4 & 2147 \\
\hline & NC-LSM & $M+30=57$ & 100.0 & 11.7 & 33.8 & 2565 \\
\hline & NC-LSM & $M+40=67$ & 100.0 & 15.0 & 40.0 & 1431 \\
\hline & LSM & $\mathrm{M}+10=37$ & 100.0 & 15.7 & 26.2 & 637 \\
\hline & LSM & $M+20=47$ & 100.0 & 18.9 & 30.0 & 879 \\
\hline & LSM & $M+30=57$ & 100.0 & 24.2 & 34.8 & 925 \\
\hline & LSM & $M+40=67$ & 100.0 & 24.0 & 41.0 & 1093 \\
\hline & P-LSM & $\mathrm{M}=27$ & 95.2 & 0.1 & 19.8 & 208 \\
\hline & P-LSM & $M+10=37$ & 100.0 & 12.1 & 25.3 & 259 \\
\hline & P-LSM & $M+20=47$ & 100.0 & 21.1 & 28.2 & 303 \\
\hline & P-LSM & $M+30=57$ & 100.0 & 24.1 & 35.9 & 620 \\
\hline & P-LSM & $M+40=67$ & 100.0 & 24.1 & 42.3 & 751 \\
\hline & R-LSM & 0 & 0 & -10.9 & 14.5 & 215 \\
\hline & R-LSM & $\mathrm{M}=27$ & 99.4 & 6.8 & 20.2 & 237 \\
\hline & R-LSM & $M+10=37$ & 99.6 & 13.0 & 25.3 & 385 \\
\hline & R-LSM & $M+20=47$ & 100.0 & 19.0 & 28.1 & 648 \\
\hline & R-LSM & $M+30=57$ & 100.0 & 23.9 & 31.9 & 888 \\
\hline & R-LSM & $M+40=67$ & 100.0 & 24.3 & 37.6 & 1113 \\
\hline \multirow[t]{23}{*}{ ecg } & ZST & $\mathrm{n} / \mathrm{a}$ & 0.0 & -29.7 & 23.3 & 944 \\
\hline & NSM & $\mathrm{n} / \mathrm{a}$ & 0.0 & -13.1 & 30.4 & 1175 \\
\hline & LSM/USM & 0 & 0.0 & -14.4 & 21.1 & 1858 \\
\hline & LSM/USM & $\mathrm{M}=15$ & 51.8 & -8.9 & 45.7 & 2329 \\
\hline & NC-LSM & $M+5=20$ & 90.6 & -2.7 & 47.6 & 2950 \\
\hline & NC-LSM & $M+10=25$ & 96.0 & 0.5 & 56.9 & 3786 \\
\hline & NC-LSM & $M+15=30$ & 97.8 & 1.9 & 65.7 & 4535 \\
\hline & NC-LSM & $M+20=35$ & 93.0 & -1.6 & 88.2 & 2966 \\
\hline & LSM & $M+5=20$ & 89.4 & -1.4 & 47.3 & 2431 \\
\hline & LSM & $M+10=25$ & 96.0 & 1.7 & 56.6 & 2798 \\
\hline & LSM & $M+15=30$ & 96.4 & 0.6 & 64.8 & 3195 \\
\hline & LSM & $M+20=35$ & 98.4 & 6.7 & 90.8 & 3643 \\
\hline & P-LSM & $\mathrm{M}=15$ & 52.2 & -8.6 & 42.7 & 9025 \\
\hline & P-LSM & $M+5=20$ & 81.4 & -4.3 & 46.1 & 10195 \\
\hline & P-LSM & $M+10=25$ & 91.8 & -1.6 & 55.9 & 10910 \\
\hline & P-LSM & $M+15=30$ & 93.4 & -1.2 & 63.8 & 11942 \\
\hline & P-LSM & $M+20=35$ & 98.6 & 5.0 & 85.3 & 14114 \\
\hline & R-LSM & 0 & 0.0 & -17.0 & 21.8 & 3569 \\
\hline & R-LSM & $\mathrm{M}=15$ & 74.8 & -4.6 & 39.4 & 3731 \\
\hline & R-LSM & $M+5=20$ & 88.6 & -2.7 & 39.6 & 3925 \\
\hline & R-LSM & $M+10=25$ & 98.0 & 3.2 & 43.8 & 4260 \\
\hline & R-LSM & $M+15=30$ & 97.8 & 4.9 & 50.2 & 4565 \\
\hline & R-LSM & $M+20=35$ & 99.8 & 9.9 & 63.3 & 4791 \\
\hline
\end{tabular}

Now, we analyze the results of ecg in Table 12. As expected, we find that the capacitive cost goes up when the safety margin is increased. The $95 \%$-slack and the yield of the R-LSM structure are monotonically increasing until $M_{\text {user }}$ is increased from $M+15=30 \mathrm{ps}$ to $M+20=35$ ps. A possible explanation for the slight decrease in $95 \%$-slack and yield is that when the safety margin $M_{u s e r}$ is increased from $M+10=25$ ps (or $M+15=30$ ps) to $M+15=30$ ps (or $M+20=35$ ps), the synthesis problem becomes more constrained and the resulting clock tree becomes substantially larger, which can be confirmed with the increase in capacitive 
cost (see Table 12). A larger clock tree is more susceptible to variations. Here, it seems that the increase in variations may have been larger than the increase in the safety margin $M_{u s e r}$. The increase in safety margin is only useful in countering the effects of variations if the increase does not enlarge the size of the clock tree to much.

\section{CONCLUSIONS}

In summary, we show that the UST-LTSM framework extends the work in [1] to handle the case when $M_{u s e r}$ is required to be greater than $M$ to increase $95 \%$-slack and yield. Moreover, the tailoring based on incrementally reducing safety margins is more effective compared to incrementally inserting safety margins.

In the future, we plan to investigate how the LTSM framework can handle designs that contain negative cycles, which result in that clusters with a large number of sinks are formed. Moreover, we will investigate the synthesis of clock networks for designs with multiple corners and multiple modes using clock scheduling and the LTSM framework.

\section{Acknowledgements}

This work was supported by NSF awards CCF-1527562 and CCF-1065318 and SRC task 292-074.

\section{9i1 REFERENCES} router for general skew constraints," ACM Transactions on Design Automation of Electronic Systems, vol. 7, no. 3, pp. 359-379, 2002.

[2] W.-C. D. Lam and C.-K. Koh, "Process variation robust clock tree routing," ASP-DAC '05, pp. 606-611, 2005.

[3] C. N. Sze, P. Restle, G.-J. Nam, and C. J. Alpert, "ISPD 2009 clock network synthesis contest," ISPD'09, pp. 149-150, 2009.

[4] C. N. Sze, "ISPD 2010 high performance clock network synthesis contest: Benchmark suite and results," ISPD'10, pp. 143-143, 2010.

[5] J. P. Fishburn, "Clock skew optimization," IEEE Transactions on Computers, vol. 39, no. 7, pp. 945-951, 1990.

[6] T. H. Cormen, C. Stein, R. L. Rivest, and C. E. Leiserson, Introduction to Algorithms. McGraw-Hill Higher Education, 2001.

[7] R. Ewetz, A. Udupa, G. Subbarayan, and C. Koh, "A TSV-cross-link-based approach to 3D-clock network synthesis for improved robustness," GLSVLSI'14, pp. 15-20, 2014.

[8] G. Ramalingam and T. Reps, "On the computational complexity of incremental algorithms," Tech. Rep. TR-1033, Computer Sciences Department, University of Wisconsin, Madison, 1991.

[9] D. Frigioni, A. Marchetti-spaccamela, and U. Nanni, "Fully dynamic shortest paths and negative cycle detection on digraphs with arbitrary arc weights," ESA'98, pp. 320-331, 1998.

[10] J. G. Xi and W. W.-M. Dai, "Useful-skew clock routing with gate sizing for low power design," DAC '96, pp. 383-388, 1996.

[11] OpenCores, "http://opencores.net/," 2014.

[12] R. Ewetz, S. Janarthanan, and C.-K. Koh, "Benchmark circuits for clock scheduling and synthesis.," https://purr.purdue.edu/publications/1759, 2015.
[13] B. V. Cherkassky and A. V. Goldberg, "Negative-cycle detection algorithms," ESA'96, pp. 349-363, 1996.

[14] Y. P. Chen and D. F. Wong, "An algorithm for zero-skew clock tree routing with buffer insertion," EDTC'96, pp. 230-237, 1996.

[15] S. Bujimalla and C.-K. Koh, "Synthesis of low power clock trees for handling power-supply variations," ISPD'11, pp. 37-44, 2011.

[16] Jonathan Richard Shewchuk, "https://www.cs.cmu.edu/quake/triangle.html," 2015.

[17] A. Agarwal, D. Blaauw, and V. Zolotov, "Statistical timing analysis for intra-die process variations with spatial correlations," ICCAD'03, pp. 900-907, 2003.

[18] M. Zhao, K. Gala, V. Zolotov, Y. Fu, R. Panda, R. Ramkumar, and B. Agrawal, "Worst case clock skew under power supply variations," TAU'02, pp. 22-28, 2002. 